\title{
A Survey on Existence Results for Boundary Value Problems of Hilfer Fractional Differential Equations and Inclusions
}

\author{
Sotiris K. Ntouyas
}

Department of Mathematics, University of Ioannina, 45110 Ioannina, Greece; sntouyas@uoi.gr

\begin{abstract}
This paper is a survey of the recent results of the author for various classes of boundary value problems for Hilfer fractional differential equations and inclusions of fractional order in $(1,2]$ supplemented with different kinds of nonlocal boundary conditions.
\end{abstract}

Keywords: Hilfer fractional derivative; Riemann-Liouville fractional derivative; Caputo fractional derivative; boundary value problems; existence; pantograph fractional differential equations

MSC: 26A33; 34A08; 34A60; 34B15

\section{Introduction}

Fractional differential equations become another necessary tool in solving real-life problems in different research areas such as mathematical biology, engineering, mechanics, and physics; for example, see the monographs [1-9]. Boundary value problems of fractional differential equations and inclusions represent an important class of applied analysis. Most researchers have studied fractional differential equations by taking Caputo or RiemannLiouville derivative. Engineers and scientists have developed some new models that involve fractional differential equations for which the Riemann-Liouville derivative is not considered appropriate. Therefore, certain modifications were introduced to avoid the difficulties and some new-type fractional order derivative operators were introduced in the literature by Hadamard, Erdelyi-Kober, Katugampola, and others. A generalization of derivatives of both Riemann-Liouville and Caputo was given by R. Hilfer in [10] and is known as the Hilfer fractional derivative of order $\alpha$ and a type $\beta \in[0,1]$, which can be reduced to the Riemann-Liouville and Caputo fractional derivatives when $\beta=0$ and $\beta=1$, respectively. Such a derivative interpolates between the Riemann-Liouville and Caputo derivative. Fractional differential equations involving Hilfer derivative have many applications; see [11-16] and references cited therein.

This survey is devoted to articles published by the author and his collaborators and concern some recent existence and uniqueness results for various classes of boundary value problems for Hilfer fractional differential equations and inclusions of fractional order in $(1,2]$ supplemented with different kinds of nonlocal boundary conditions.

The rest of this survey is organized as follows. In Section 2, we introduce some notations and definitions of fractional calculus and multivalued analysis. In the subsequent sections, we present existence and uniqueness results for boundary value problems for Hilfer, $\psi$-Hilfer fractional, and sequential fractional differential equations and inclusions with a variety of nonlocal boundary conditions, such as multipoint, integral, integral multipoint, integro-multipoint, integro-multistrip-multipoint and Riemann-Stieltjes integral multistrip. We also present existence and uniqueness results for coupled systems of Hilfer and $\psi$-Hilfer types and Hilfer-Hadamard fractional and sequential fractional differential equations. Note that our goal here is a more complete and comprehensive review, and as such, the choice is made to include as many results as possible to illustrate the progress on the matter. Any proofs (that are rather long) are omitted, for this matter, and the reader is referred to the relative article accordingly. 
Let us describe briefly the used methods to obtain our results. In each problem, we first present an auxiliary result concerning a linear variant of the corresponding boundary value problem that is very useful to transform the studied problem into a fixed point problem. A variety of fixed point theorems are then used to establish the existence and uniqueness results. For the single-valued case, the fixed point theorems of Banach, Boyd and Wong, Krasnoselskii, Sadovskii, Isaia and the Leray-Schauder nonlinear alternative were used, while in the multivalued case, the fixed point theorems of Bohnenblust-Karlin, Martelli, Covitz-Nadler and the Leray-Schauder nonlinear alternative for multivalued maps maps were used. For the multivalued case, we present existence results for both cases, convex-valued (upper semicontinuous case), and nonconvex-valued (Lipschitz case) multifunctions. To obtain the existence and uniqueness results for fractional coupled systems, Banach's contraction mapping principle and the Leray-Schauder alternative are used. In each theorem, we indicate the used fixed point theorem.

\section{Preliminaries}

In this section, we introduce some notations and definitions of fractional calculus and multivalued analysis.

\subsection{Fractional Calculus}

Let $C([a, b], \mathbb{R})$ denote the Banach space of all continuous functions from $[a, b]$ to $\mathbb{R}$ endowed with the norm defined by $\|x\|=\sup _{t \in[a, b]}|x(t)|$. It is obvious that the product space $(C([a, b], \mathbb{R}) \times C([a, b], \mathbb{R}),\|(x, y)\|)$ is Banach space with the norm $\|(x, y)\|=\|x\|+$ $\|y\| . A C^{n}([c, d], \mathbb{R})$ is the $n$-times absolutely continuous functions defined as

$$
A C^{n}([c, d], \mathbb{R})=\left\{f:[c, d] \longrightarrow \mathbb{R} ; f^{(n-1)} \in A C([c, d], \mathbb{R})\right\} .
$$

Definition 1. The Riemann-Liouville fractional integral of order $\alpha>0$ of a continuous function $u:[a, \infty) \rightarrow \mathbb{R}$ is defined by

$$
I^{\alpha} u(t)=\frac{1}{\Gamma(\alpha)} \int_{a}^{t}(t-s)^{\alpha-1} u(s) d s,
$$

provided the right-hand side exists on $(a, \infty)$.

Definition 2. The Riemann-Liouville fractional derivative of order $\alpha>0$ of a continuous function $u$ is defined by

$$
{ }^{R L} D^{\alpha} u(t):=D^{n} I^{n-\alpha} u(t)=\frac{1}{\Gamma(n-\alpha)}\left(\frac{d}{d t}\right)^{n} \int_{a}^{t}(t-s)^{n-\alpha-1} u(s) d s,
$$

where $n=[\alpha]+1,[\alpha]$ denotes the integer part of real number $\alpha$, provided the right-hand side is pointwise defined on $(a, \infty)$.

Definition 3. The Caputo fractional derivative of order $\alpha>0$ of a continuous function $u$ is defined as

$$
{ }^{C} D^{\alpha} u(t):=I^{n-\alpha} D^{n} u(t)=\frac{1}{\Gamma(n-\alpha)} \int_{a}^{t}(t-s)^{n-\alpha-1}\left(\frac{d}{d s}\right)^{n} u(s) d s, n-1<\alpha<n,
$$

provided the right-hand side is pointwise defined on $(a, \infty)$.

In [10] (see also [11]), another new definition of the fractional derivative, known as the generalized Riemann-Liouville fractional derivative, was suggested; it is defined as 
Definition 4. The generalized Riemann-Liouville fractional derivative or Hilfer fractional derivative of order $\alpha$ and parameter $\beta$ of a function $u$ is defined by

$$
{ }^{H} D^{\alpha, \beta} u(t)=I^{\beta(n-\alpha)} D^{n} I^{(1-\beta)(n-\alpha)} u(t),
$$

where $n-1<\alpha<n, 0 \leq \beta \leq 1, t>a, D^{n}=\frac{d^{n}}{d t^{n}}$.

Remark 1. In the above definition, type $\beta$ allows $D^{\alpha, \beta}$ to interpolate continuously between the classical Riemann-Liouville fractional derivative and the Caputo fractional derivative. When $\beta=0$, the Hilfer fractional derivative corresponds to the Riemann-Liouville fractional derivative

$$
{ }^{H} D^{\alpha, 0} u(t)=D^{n} I^{n-\alpha} u(t),
$$

while when $\beta=1$, the Hilfer fractional derivative corresponds to the Caputo fractional derivative

$$
{ }^{H} D^{\alpha, 1} u(t)=I^{n-\alpha} D^{n} u(t) .
$$

In the following, we recall some notations and results from $\psi$-Hilfer fractional derivatives.

Definition 5 ([2]). Let $(a, b),(-\infty \leq a<b \leq \infty)$ be a finite or infinite interval of the half-axis $(0, \infty)$ and $\alpha>0$. In addition, let $\psi(t)$ be a positive increasing function on $(a, b]$, which has $a$ continuous derivative $\psi^{\prime}(t)$ on $(a, b)$. The $\psi$-Riemann-Liouville fractional integral of a function $f$ with respect to another function $\psi$ on $[a, b]$ is defined by

$$
I_{a^{+}}^{\alpha ; \psi} f(t)=\frac{1}{\Gamma(\alpha)} \int_{a}^{t} \psi^{\prime}(s)(\psi(t)-\psi(s))^{\alpha-1} f(s) d s, \quad t>a>0,
$$

where $\Gamma(\cdot)$ represents the Gamma function.

Definition 6 ([2]). Let $\psi^{\prime}(t) \neq 0$ and $\alpha>0, n \in \mathbb{N}$. The Riemann-Liouville derivatives of $a$ function $f$ with respect to another function $\psi$ of order $\alpha$ correspondent to the Riemann-Liouville is defined by

$$
\begin{aligned}
D_{a^{+}}^{\alpha ; \psi} f(t) & =\left(\frac{1}{\psi^{\prime}(t)} \frac{d}{d t}\right)^{n} I_{a^{+}}^{n-\alpha ; \psi} f(t) \\
& =\frac{1}{\Gamma(n-\alpha)}\left(\frac{1}{\psi^{\prime}(t)} \frac{d}{d t}\right)^{n} \int_{a}^{t} \psi^{\prime}(s)(\psi(t)-\psi(s))^{n-\alpha-1} f(s) d s,
\end{aligned}
$$

where $n=[\alpha]+1,[\alpha]$ represents the integer part of the real number $\alpha$. This is the greatest integer $n$ such that $n \leq \alpha$.

Definition 7 ([17]). Let $n-1<\alpha<n$ with $n \in \mathbb{N},[a, b]$ is the interval such that $-\infty \leq a<$ $b \leq \infty$ and $f, \psi \in C^{n}([a, b], \mathbb{R})$ two functions such that $\psi$ is increasing, and $\psi^{\prime}(t) \neq 0$ for all $t \in[a, b]$. The $\psi$-Hilfer fractional derivative of a function $f$ of order $\alpha$ and type $0 \leq \beta \leq 1$ is defined by

$$
{ }^{H} D_{a^{+}}^{\alpha, \beta ; \psi} f(t)=I_{a^{+}}^{\beta(n-\alpha) ; \psi}\left(\frac{1}{\psi^{\prime}(t)} \frac{d}{d t}\right)^{n} I_{a^{+}}^{(1-\beta)(n-\alpha) ; \psi} f(t)=I_{a^{+}}^{\gamma-\alpha ; \psi} D_{a^{+}}^{\gamma ; \psi} f(t),
$$

where $n=[\alpha]+1,[\alpha]$ represents the integer part of the real number $\alpha$ with $\gamma=\alpha+\beta(n-\alpha)$.

Some preliminaries from the Hilfer-Hadamard fractional derivative are presented next. 
Definition 8 (Hadamard fractional integral [2]). The Hadamard fractional integral of order $\alpha \in \mathbb{R}_{+}$for a function $f:[a, \infty) \rightarrow \mathbb{R}$ is defined as

$$
{ }_{H} I_{a^{+}}^{\alpha} f(t)=\frac{1}{\Gamma(\alpha)} \int_{a}^{t}\left(\log \frac{t}{\tau}\right)^{\alpha-1} \frac{f(\tau)}{\tau} d \tau, \quad(t>a)
$$

provided the integral exists, where $\log (\cdot)=\log _{e}(\cdot)$.

Definition 9 (Hadamard fractional derivative [2]). The Hadamard fractional derivative of order $\alpha>0$ applied to the function $f:[a, \infty) \rightarrow \mathbb{R}$ is defined as

$$
{ }_{H} D_{a^{+}}^{\alpha} f(t)=\delta^{n}\left({ }_{H} I_{a^{+}}^{n-\alpha} f(t)\right), \quad n-1<\alpha<n, \quad n=[\alpha]+1,
$$

where $\delta^{n}=\left(t \frac{d}{d t}\right)^{n}$ and $[\alpha]$ denotes the integer part of the real number $\alpha$.

The Hilfer-Hadamard fractional derivative may be viewed as interpolating the Hadamard fractional derivative. Indeed, for $\beta=0$, this derivative reduces to the Hadamard fractional derivative.

Definition 10 (Hilfer-Hadamard fractional derivative [18]). Let $n-1<\alpha<n$ and $0 \leq \beta \leq$ $1, f \in L^{1}(a, b)$. The Hilfer-Hadamard fractional derivative of order $\alpha$ and type $\beta$ of $f$ is defined as

$$
\begin{aligned}
\left({ }_{H} D_{a^{+}}^{\alpha, \beta} f\right)(t) & =\left({ }_{H} I_{a^{+}}^{\beta(n-\alpha)} \delta^{n}{ }_{H} I_{a^{+}}^{(n-\alpha)(1-\beta)} f\right)(t) \\
& =\left({ }_{H} I_{a^{+}}^{\beta(n-\alpha)} \delta^{n}{ }_{H} I_{a^{+}}^{n-\gamma} f\right)(t) ; \gamma=\alpha+n \beta-\alpha \beta \\
& =\left({ }_{H} I_{a^{+}}^{\beta(n-\alpha)}{ }_{H} D_{a^{+}}^{\gamma} f\right)(t),
\end{aligned}
$$

where $I_{a^{+}}^{(.)}$and ${ }_{H} D_{a^{+}}^{(.)}$are the Hadamard fractional integral and derivative defined by (1) and(2), respectively.

\subsection{Multivalued Analysis}

For a normed space $(X,\|\cdot\|)$, we define: $\mathcal{P}(X)=\{Y \subset X: Y \neq \varnothing\} ; \mathcal{P}_{c p}(X)=\{Y \in$ $\mathcal{P}(X): Y$ is compact $\} ; \mathcal{P}_{c, c p}(X)=\{Y \in \mathcal{P}(X): Y$ is convex and compact $\} ; \mathcal{P}_{b, c l}(X)=$ $\{Y \in \mathcal{P}(X): Y$ is bounded and closed $\} ; \mathcal{P}_{b, c l, c}(X)=\{Y \in \mathcal{P}(X): Y$ is bounded, closed and convex $\}$ and $\mathcal{P}_{c l}(X)=\{Y \in \mathcal{P}(X): Y$ is closed $\}$.

For the basic concepts of multivalued analysis, we refer to $[19,20]$.

A multivalued map $G: X \rightarrow \mathcal{P}(X)$ has a fixed point if there is $x \in X$ such that $x \in G(x)$.

Definition 11. A multivalued map $F:[a, b] \times \mathbb{R} \rightarrow \mathcal{P}(\mathbb{R})$ is said to be Carathéodory if:

(i) $t \longmapsto F(t, x)$ is measurable for each $x \in \mathbb{R}$;

(ii) $x \longmapsto F(t, x)$ is upper semicontinuous for almost all $t \in[a, b]$.

Furthermore, a Carathéodory function $F$ is called $L^{1}$-Carathéodory if:

(iii) for each $\rho>0$, there exists $\varphi_{\rho} \in L^{1}\left([a, b], \mathbb{R}^{+}\right)$such that

$$
\|F(t, x)\|=\sup \{|v|: v \in F(t, x)\} \leq \varphi_{\rho}(t)
$$

for all $x \in \mathbb{R}$ with $\|x\| \leq \rho$ and for a.e. $t \in[a, b]$. 


\section{Boundary Value Problems for Hilfer Fractional Differential Equations and Inclusions}

3.1. Nonlocal Fractional Integral Boundary Conditions

The study of boundary value problems for Hilfer-type fractional differential equations of order $\alpha \in(1,2]$ with nonlocal integral boundary conditions, initiated in [21], is conducted by considering the following problem

$$
\left\{\begin{array}{l}
{ }^{H} D^{\alpha, \beta} x(t)=f(t, x(t)), \quad t \in[a, b] \\
x(a)=0, \quad x(b)=\sum_{i=1}^{m} \delta_{i} I^{\varphi_{i}} x\left(\xi_{i}\right)
\end{array}\right.
$$

where ${ }^{H} D^{\alpha, \beta}$ is the Hilfer fractional derivative of order $\alpha, 1<\alpha<2$, and parameter $\beta$, $0 \leq \beta \leq 1$, and $I^{\varphi_{i}}$ is the Riemann-Liouville fractional integral of order $\varphi_{i}>0, \xi_{i} \in[a, b]$, $a \geq 0$, and $\delta_{i} \in \mathbb{R}$.

The following lemma deals with a linear variant of the boundary value problem (3).

Lemma 1. Let $\varphi_{i}>0, \Lambda \neq 0, \xi_{i} \in[a, b], a \geq 0, \delta_{i} \in \mathbb{R}, 1<\alpha<2, \gamma=\alpha+2 \beta-\alpha \beta$ and $h \in C([a, b], \mathbb{R})$. Then, the function $x$ is a solution of the boundary value

$$
\left\{\begin{array}{l}
{ }^{H} D^{\alpha, \beta} x(t)=h(t), \quad t \in[a, b], \\
x(a)=0, \quad x(b)=\sum_{i=1}^{m} \delta_{i} I^{\varphi_{i}} x\left(\xi_{i}\right),
\end{array}\right.
$$

if and only if

$$
x(t)=\frac{(t-a)^{\gamma-1}}{\Lambda \Gamma(\gamma)}\left(I^{\alpha} h(b)-\sum_{i=1}^{m} \delta_{i} I^{\alpha+\varphi_{i}} h\left(\xi_{i}\right)\right)+I^{\alpha} h(t)
$$

where

$$
\Lambda=\sum_{i=1}^{m} \frac{\delta_{i}\left(\xi_{i}-a\right)^{\gamma+\varphi_{i}-1}}{\Gamma\left(\gamma+\varphi_{i}\right)}-\frac{(b-a)^{\gamma-1}}{\Gamma(\gamma)}
$$

In view of Lemma 1 , we define an operator $\mathcal{A}: C([a, b], \mathbb{R}) \rightarrow C([a, b], \mathbb{R})$ by

$$
\begin{aligned}
(\mathcal{A} x)(t)= & \frac{(t-a)^{\gamma-1}}{\Lambda \Gamma(\gamma)}\left(I^{\alpha} f(s, x(s))(b)-\sum_{i=1}^{m} \delta_{i} I^{\alpha+\varphi_{i}} f(s, x(s))\left(\xi_{i}\right)\right) \\
& +I^{\alpha} f(s, x(s))(t) .
\end{aligned}
$$

It should be noticed that problem (3) has solutions if and only if the operator $\mathcal{A}$ has fixed points.

\subsubsection{Existence and Uniqueness Results for the Problem (3)}

The existence and uniqueness results for the boundary value problem (3) are given in the following theorems. Theorem 1 is based on Banach's contraction mapping principle, Theorem 2 on Banach contraction mapping principle together with Hölder inequality, and Theorem 3 on Boyd and Wong fixed point theorem for nonlinear contractions.

Theorem 1. Assume that:

(1.1) There exists a constant $L>0$ such that $|f(t, x)-f(t, y)| \leq L|x-y|$ for each $t \in[a, b]$ and $x, y \in \mathbb{R}$.

Then, the boundary value problem (3) has a unique solution on $[a, b]$, provided that

$$
L\left\{\frac{(b-a)^{\alpha}}{\Gamma(\alpha+1)}+\frac{(b-a)^{\gamma-1}}{|\Lambda| \Gamma(\gamma)}\left(\frac{(b-a)^{\alpha}}{\Gamma(\alpha+1)}+\sum_{i=1}^{m}\left|\delta_{i}\right| \frac{\left(\xi_{i}-a\right)^{\alpha+\varphi_{i}}}{\Gamma\left(\alpha+\varphi_{i}+1\right)}\right)\right\}<1 .
$$


Theorem 2. Suppose that $f:[a, b] \times \mathbb{R} \rightarrow \mathbb{R}$ is a continuous function. In addition, we assume that:

(2.1) $|f(t, x)-f(t, y)| \leq \theta(t)|x-y|$, for $t \in[a, b], x, y \in \mathbb{R}$ and $\theta \in L^{1 / \sigma}\left([a, b], \mathbb{R}^{+}\right), \sigma \in$ $(0,1)$.

If $\|\theta\| \omega<1$, then the boundary value problem (3) has a unique solution on $[a, b]$, where $\|\theta\|=\left(\int_{a}^{b}|\theta(s)|^{1 / \sigma} d s\right)^{\sigma}$ and

$$
\begin{aligned}
\omega= & \frac{(b-a)^{\gamma+\alpha-\sigma-1}}{|\Lambda| \Gamma(\gamma) \Gamma(\alpha)}\left(\frac{1-\sigma}{\alpha-\sigma}\right)^{1-\sigma}+\frac{(b-a)^{\gamma-1}}{|\Lambda| \Gamma(\gamma)} \sum_{i=1}^{m} \frac{\left|\delta_{i}\right|\left(\xi_{i}-a\right)^{\alpha+\varphi_{i}-\sigma}}{\Gamma(\alpha+\varphi)}\left(\frac{1-\sigma}{\alpha+\varphi_{i}-\sigma}\right)^{1-\sigma} \\
& +\frac{(b-a)^{\alpha-\sigma}}{\Gamma(\alpha)}\left(\frac{1-\sigma}{\alpha-\sigma}\right)^{1-\sigma} .
\end{aligned}
$$

Theorem 3. Let $f:[a, b] \times \mathbb{R} \rightarrow \mathbb{R}$ be a continuous function satisfying the assumption:

$|f(t, x)-f(t, y)| \leq h(t) \frac{|x-y|}{H^{*}+|x-y|}$, for $t \in[a, b], x, y \geq 0$, where $h:[a, b] \rightarrow \mathbb{R}^{+}$is continuous and $H^{*}$ the constant defined by

$$
H^{*}:=\frac{(b-a)^{\gamma-1}}{|\Lambda| \Gamma(\gamma)} I^{\alpha} h(b)+\frac{(b-a)^{\gamma-1}}{|\Lambda| \Gamma(\gamma)} \sum_{i=1}^{m}\left|\delta_{i}\right| I^{\alpha+\varphi_{i}} h\left(\xi_{i}\right)+I^{\alpha} h(b)
$$

Then, the boundary value problem (3) has a unique solution on $[a, b]$.

Two existence results are presented now, based on Krasnoselskii's fixed point theorem and the Leray-Schauder nonlinear alternative, respectively.

Theorem 4. Let $f:[a, b] \times \mathbb{R} \rightarrow \mathbb{R}$ be a continuous function satisfying (1.1). In addition, we assume that:

(4.1) $|f(t, x)| \leq \varphi(t), \quad \forall(t, x) \in[a, b] \times \mathbb{R}$, and $\varphi \in C\left([a, b], \mathbb{R}^{+}\right)$.

Then, the boundary value problem (3) has at least one solution on $[a, b]$ provided that

$$
L \frac{(b-a)^{\gamma-1}}{|\Lambda| \Gamma(\gamma)}\left(\frac{(b-a)^{\alpha}}{\Gamma(\alpha+1)}+\sum_{i=1}^{m}\left|\delta_{i}\right| \frac{\left(\xi_{i}-a\right)^{\alpha+\varphi_{i}}}{\Gamma\left(\alpha+\varphi_{i}+1\right)}\right)<1
$$

Theorem 5. Assume that:

(5.1) there exists a continuous nondecreasing function $\psi:[0, \infty) \rightarrow(0, \infty)$ and a function $p \in C\left([a, b], \mathbb{R}^{+}\right)$such that

$$
|f(t, u)| \leq p(t) \psi(\|x\|) \quad \text { for each } \quad(t, x) \in[a, b] \times \mathbb{R}
$$

(5.2) there exists a constant $M>0$ such that

$$
\frac{M}{\psi(M)\|p\|\left\{\frac{(b-a)^{\alpha}}{\Gamma(\alpha+1)}+\frac{(b-a)^{\gamma-1}}{|\Lambda| \Gamma(\gamma)}\left(\frac{(b-a)^{\alpha}}{\Gamma(\alpha+1)}+\sum_{i=1}^{m}\left|\delta_{i}\right| \frac{\left(\xi_{i}-a\right)^{\alpha+\varphi_{i}}}{\Gamma\left(\alpha+\varphi_{i}+1\right)}\right)\right\}}>1 .
$$

Then, the boundary value problem (3) has at least one solution on $[a, b]$. 
3.1.2. Existence Results for the Inclusion Problem

The multivalued case of the problem (3), that is, the boundary value problem of Hilfer-type fractional differential inclusions with nonlocal integral boundary conditions

$$
\left\{\begin{array}{c}
{ }^{H} D^{\alpha, \beta} x(t) \in F(t, x(t)), \quad t \in[a, b], \\
x(a)=0, \quad x(b)=\sum_{i=1}^{m} \delta_{i} I^{\varphi_{i}} x\left(\xi_{i}\right),
\end{array}\right.
$$

were studied in [22], where $F:[a, b] \times \mathbb{R} \rightarrow \mathcal{P}(\mathbb{R})$ is a multivalued map, $\mathcal{P}(\mathbb{R})$ is the family of all nonempty subsets of $\mathbb{R}$, and the other parameters are as in problem (3).

Definition 12. A function $x \in A C([a, b], \mathbb{R})$ is said to be a solution of the problem (4) if there exists a function $v \in L^{1}([a, b], \mathbb{R})$ with $v(t) \in F(t, x)$ for a.e. $t \in[a, b]$ such that $x$ satisfies the differential equation ${ }^{H} D^{\alpha, \beta} x(t)=v(t)$ on $[a, b]$ and the boundary conditions $x(a)=0, x(b)=$ $\sum_{i=1}^{m} \delta_{i} I^{\varphi_{i}} x\left(\xi_{i}\right)$

Our existence results for convex- and nonconvex-valued multifunctions, based, respectively, on the Leray-Schauder nonlinear alternative for multivalued maps maps and Covitz and Nadler fixed point theorem for contractive multivalued maps, are as follows.

Theorem 6. Assume that (5.2) holds. In addition, we suppose that:

(6.1) $\quad F:[a, b] \times \mathbb{R} \rightarrow \mathcal{P}_{c, c p}(\mathbb{R})$ is $L^{1}$-Carathéodory;

(6.2) there exists a continuous nondecreasing function $\psi:[0, \infty) \rightarrow(0, \infty)$ and a function $p \in C\left([a, b], \mathbb{R}^{+}\right)$such that

$$
\|F(t, x)\|_{\mathcal{P}}:=\sup \{|x|: x \in F(t, x)\} \leq p(t) \psi(\|x\|) \text { for each }(t, x) \in[a, b] \times \mathbb{R} .
$$

Then, the boundary value problem (4) has at least one solution on $[a, b]$.

Theorem 7. Assume that the following conditions hold:

(7.1) $\quad F:[a, b] \times \mathbb{R} \rightarrow \mathcal{P}_{c p}(\mathbb{R})$ is such that $F(\cdot, x):[a, b] \rightarrow \mathcal{P}_{c p}(\mathbb{R})$ is measurable for each $x \in \mathbb{R}$;

(7.2) $\quad H_{d}(F(t, x), F(t, \bar{x})) \leq m(t)|x-\bar{x}|$ for almost all $t \in[a, b]$ and $x, \bar{x} \in \mathbb{R}$ with $m \in$ $C\left([a, b], \mathbb{R}^{+}\right)$and $d(0, F(t, 0)) \leq m(t)$ for almost all $t \in[a, b]$.

Then, the boundary value problem (4) has at least one solution on $[a, b]$ if

$$
\left\{\frac{(b-a)^{\alpha}}{\Gamma(\alpha+1)}+\frac{(b-a)^{\gamma-1}}{|\Lambda| \Gamma(\gamma)}\left(\frac{(b-a)^{\alpha}}{\Gamma(\alpha+1)}+\sum_{i=1}^{m}\left|\delta_{i}\right| \frac{\left(\xi_{i}-a\right)^{\alpha+\varphi_{i}}}{\Gamma\left(\alpha+\varphi_{i}+1\right)}\right)\right\}\|m\|<1 .
$$

3.2. Pantograph Fractional Differential Equations and Inclusions with Nonlocal Fractional Integral Boundary Conditions

A new class of boundary value problems of pantograph equations with Hilfer-type fractional differential equations and nonlocal integral boundary conditions of the form

$$
\left\{\begin{array}{l}
{ }^{H} D^{\alpha, \beta} x(t)=f(t, x(t), x(\lambda t)), \quad t \in[a, b], \\
x(a)=0, \quad A x(b)+B I^{\delta} x(\eta)=c, \quad \eta \in(a, b),
\end{array}\right.
$$

were introduced in [23], where ${ }^{H} D^{\alpha, \beta}$ is the Hilfer fractional derivative of order $\alpha, 1<\alpha<2$, and parameter $\beta, 0 \leq \beta \leq 1, f:[a, b] \times \mathbb{R} \times \mathbb{R} \rightarrow \mathbb{R}$ is a continuous function, $I^{\delta}$ is the Riemann-Liouville fractional integral of order $\delta>0, a \geq 0, A, B, c \in \mathbb{R}$, and $0<\lambda<1$.

The following lemma deals with a linear variant of the boundary value problem (5). 
Lemma 2. Let $a \geq 0,1<\alpha<2, \gamma=\alpha+2 \beta-\alpha \beta, h \in C([a, b], \mathbb{R})$, and

$$
\Lambda:=\frac{A(b-a)^{\gamma-1}}{\Gamma(\gamma)}+\frac{B(\eta-a)^{\gamma+\delta-1}}{\Gamma(\gamma+\delta)} \neq 0 .
$$

Then, the function $x$ is a solution of the boundary value problem

$$
\left\{\begin{array}{l}
{ }^{H} D^{\alpha, \beta} x(t)=h(t), \quad t \in[a, b], \\
x(a)=0, \quad A x(b)+B I^{\delta} x(\eta)=c, \eta \in(a, b),
\end{array}\right.
$$

if and only if

$$
x(t)=I^{\alpha} h(t)+\frac{(t-a)^{\gamma-1}}{\Lambda \Gamma(\gamma)}\left[c-A I^{\alpha} h(b)-B I^{\alpha+\delta} h(\eta)\right] .
$$

In view of Lemma 2 , we define an operator $\mathcal{A}: C([a, b], \mathbb{R}) \rightarrow C([a, b], \mathbb{R})$ by

$$
\begin{aligned}
(\mathcal{A} x)(t)= & \frac{(t-a)^{\gamma-1}}{\Lambda \Gamma(\gamma)}\left(c-A I^{\alpha} f(s, x(s), x(\lambda s))(b)\right. \\
& \left.-B I^{\alpha+\delta} f(s, x(s), x(\lambda s))(\eta)\right)+I^{\alpha} f(s, x(s), x(\lambda s))(t) .
\end{aligned}
$$

It should be noticed that problem (5) has solutions if and only if the operator $\mathcal{A}$ has fixed points.

3.2.1. Existence and Uniqueness Results for the Problem (5)

The existence and uniqueness result for the problem (5), based on Banach's contraction mapping principle, is as follows.

Theorem 8. Assume that:

(8.1) there exists a constant $L>0$ such that

$$
\left|f\left(t, x_{1}, x_{2}\right)-f\left(t, y_{1}, y_{2}\right)\right| \leq L\left(\left|x_{1}-y_{1}\right|+\left|x_{2}-y_{2}\right|\right)
$$

for each $t \in[a, b]$ and $x_{i}, y_{i} \in \mathbb{R}, i=1,2$.

If

$$
2 L\left\{\frac{(b-a)^{\gamma-1}}{|\Lambda| \Gamma(\gamma)}\left[|A| \frac{(b-a)^{\alpha}}{\Gamma(\alpha+1)}+|B| \frac{(\eta-a)^{\alpha+\delta}}{\Gamma(\alpha+\delta+1)}\right]+\frac{(b-a)^{\alpha}}{\Gamma(\alpha+1)}\right\}<1,
$$

then the boundary value problem (5) has a unique solution on $[a, b]$.

The existence results, based on Krasnoselskii's fixed point theorem and the LeraySchauder nonlinear alternative, respectively, are given in the following theorems.

Theorem 9. Let $f:[a, b] \times \mathbb{R} \times \mathbb{R} \rightarrow \mathbb{R}$ be a continuous function satisfying (8.1). In addition, we assume that:

(9.1) $|f(t, x, y)| \leq \varphi(t), \quad \forall(t, x, y) \in[a, b] \times \mathbb{R} \times \mathbb{R}$, and $\varphi \in C\left([a, b], \mathbb{R}^{+}\right)$.

Then, the boundary value problem (5) has at least one solution on $[a, b]$, provided

$$
L \frac{(b-a)^{\gamma-1}}{|\Lambda| \Gamma(\gamma)}\left[|B| \frac{(\eta-a)^{\alpha+\delta}}{\Gamma(\alpha+\delta+1)}+|A| \frac{(b-a)^{\alpha}}{\Gamma(\alpha+1)}\right]<1
$$

Theorem 10. Assume that: 
(10.1) there exist a continuous nondecreasing function $\psi:[0, \infty) \rightarrow(0, \infty)$ and a function $p \in C\left([a, b], \mathbb{R}^{+}\right)$such that

$$
|f(t, u, v)| \leq p(t) \psi(|u|+|v|) \quad \text { for each } \quad(t, u, v) \in[a, b] \times \mathbb{R} \times \mathbb{R} ;
$$

(10.2) there exists a constant $M>0$ such that

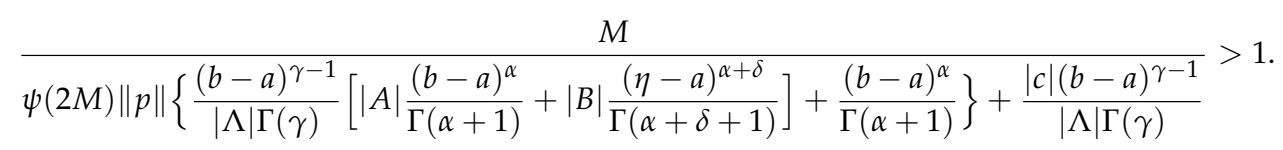

Then, the boundary value problem (5) has at least one solution on $[a, b]$.

\subsubsection{Existence Results for the Inclusion Problem}

The multivalued version of the problem (5) were studied also in [23] by considering the following inclusion problem

$$
\left\{\begin{array}{l}
{ }^{H} D^{\alpha, \beta} x(t) \in F(t, x(t), x(\lambda t)), \quad t \in[a, b], \\
x(a)=0, \quad A x(b)+B I^{\delta} x(\eta)=c, \quad \eta \in(a, b),
\end{array}\right.
$$

where $F:[a, b] \times \mathbb{R} \times \mathbb{R} \rightarrow \mathcal{P}(\mathbb{R})$ is a multivalued function and $(\mathcal{P}(\mathbb{R})$ is the family of all nonempty subsets of $\mathbb{R})$.

Definition 13. A function $x \in A C([a, b], \mathbb{R})$ is said to be a solution of the problem (6) if $x(a)=$ $0, A x(b)+B I^{\delta} x(\eta)=c$, and there exists a function $v \in L^{1}([a, b], \mathbb{R})$ with $v \in F(t, x, y)$ a.e. on $[a, b]$ such that

$$
x(t)=\frac{(t-a)^{\gamma-1}}{\Lambda \Gamma(\gamma)}\left(c-A I^{\alpha} v(s)(b)-B I^{\alpha+\delta} v(s)(\eta)\right)+I^{\alpha} v(s)(t), \quad t \in[a, b] .
$$

The existence results in the case when $F$ has convex values (the upper semicontinuous case) are given in the next theorems. Theorem 11 is based on the Bohnenblust-Karlin fixed point theorem, Theorem 12 on Martelli's fixed point theorem, and Theorem 13 on the Leray-Schauder nonlinear alternative for multivalued maps.

Theorem 11. Assume that:

(11.1) $F:[a, b] \times \mathbb{R} \times \mathbb{R} \rightarrow \mathcal{P}_{c, c p}(\mathbb{R})$ is $L^{1}$-Carathéodory;

(11.2) $\liminf _{\rho \rightarrow \infty} \frac{1}{\rho} \int_{a}^{b} \phi_{\rho}(t) d t=\mu$, where $\varphi_{\rho}$ is the function that appears in Definition 11.

Then, the boundary problem (6) has at least one solution on $[a, b]$, provided that:

$$
\left\{\frac{(b-a)^{\gamma-1}}{|\Lambda| \Gamma(\gamma)}\left(|A| \frac{b^{\alpha-1}}{\Gamma(\alpha)}+|B| \frac{\eta^{\alpha+\delta-1}}{\Gamma(\alpha+\delta)}\right)+\frac{b^{\alpha-1}}{\Gamma(\alpha)}\right\} \mu<1
$$

Theorem 12. Assume that the following hypotheses hold:

(12.1) $F:[a, b] \times \mathbb{R} \times \mathbb{R} \rightarrow \mathcal{P}_{b, c l, c}(\mathbb{R})$ is a $L^{1}$-Carathéodory multivalued map;

(12.2) there exists a function $h \in C([a, b], \mathbb{R})$ such that

$$
\|F(t, x, y)\| \leq h(t), \text { for a.e. } t \in[a, b] \text { and each } x, y \in \mathbb{R} .
$$

Then, the problem (6) has at least one solution on $[a, b]$.

Theorem 13. Assume that (10.2) and (11.1) hold. In addition, we assume that:

(13.1) there exists a continuous nondecreasing function $\psi:[0, \infty) \rightarrow(0, \infty)$ and a function $p \in C\left([a, b], \mathbb{R}^{+}\right)$such that 
$\|F(t, x, y)\|_{\mathcal{P}}:=\sup \{|v|: v \in F(t, x, y)\} \leq p(t) \psi(|x|)$ for each $(t, x, y) \in[a, b] \times \mathbb{R} \times$ $\mathbb{R}$.

Then, the boundary value problem (6) has at least one solution on $[a, b]$.

Now, we state the existence of solutions for the boundary value problem (6) with a nonconvex-valued right hand side. The proof is based on the Covitz and Nadler fixed point theorem.

Theorem 14. Assume that the following conditions hold:

(14.1) $F:[a, b] \times \mathbb{R} \times \mathbb{R} \rightarrow \mathcal{P}_{c p}(\mathbb{R})$ is such that $F(\cdot, x, y):[a, b] \rightarrow \mathcal{P}_{c p}(\mathbb{R})$ is measurable for each $x, y \in \mathbb{R}$;

(14.2) $H_{d}(F(t, x, y), F(t, \bar{x}), \bar{y}) \leq m(t)(|x-\bar{x}|+|y-\bar{y}|)$ for almost all $t \in[a, b]$ and $x, \bar{x}, y, \bar{y} \in$ $\mathbb{R}$ with $m \in C\left([a, b], \mathbb{R}^{+}\right)$and $d(0, F(t, 0,0)) \leq m(t)$ for almost all $t \in[a, b]$.

Then, the boundary value problem (6) has at least one solution on $[a, b]$ if

$$
2\left(\frac{(b-a)^{\gamma-1}}{|\Lambda| \Gamma(\gamma)}\left[|B| \frac{(\eta-a)^{\alpha+\delta}}{\Gamma(\alpha+\delta+1)}+|A| \frac{(b-a)^{\alpha}}{\Gamma(\alpha+1)}\right]+\frac{(b-a)^{\alpha}}{\Gamma(\alpha+1)}\right)\|m\|<1
$$

\subsection{Nonlocal Integro-Multipoint Boundary Conditions}

Existence and uniqueness of solutions for a new class of boundary value problems of Hilfer-type fractional differential equations with nonlocal integro-multipoint boundary conditions of the form

$$
\left\{\begin{array}{l}
{ }^{H} D^{\alpha, \beta} x(t)=f\left(t, x(t), I^{\delta} x(t)\right), \quad t \in[a, b], \\
x(a)=0, \quad \int_{a}^{b} x(s) d s+\mu=\sum_{i=1}^{m-2} \zeta_{i} x\left(\theta_{i}\right),
\end{array}\right.
$$

were studied in [24], where ${ }^{H} D^{\alpha, \beta}$ is the Hilfer fractional derivative of order $\alpha, 1<\alpha<2$, and parameter $\beta, 0 \leq \beta \leq 1, f:[a, b] \times \mathbb{R} \times \mathbb{R} \rightarrow \mathbb{R}$ is a continuous function, $I^{\delta}$ is the Riemann-Liouville fractional integral of order $\delta>0$, and the points $a<\theta_{1}<\theta_{2}<\cdots<$ $\theta_{m-2}<b, a \geq 0$, and $\mu, \zeta_{i} \in \mathbb{R}, i=1,2, \ldots, m-2$ are given constants.

The following lemma deals with a linear variant of the boundary value problem (7).

Lemma 3. Let $a \geq 0,1<\alpha<2, \gamma=\alpha+2 \beta-\alpha \beta, h \in C([a, b], \mathbb{R})$ and

$$
\Lambda:=\frac{(b-a)^{\gamma}}{\gamma}-\sum_{i=1}^{m-2} \zeta_{i}\left(\theta_{i}-a\right)^{\gamma-1} \neq 0 .
$$

Then, the function $x \in C([a, b], \mathbb{R})$ is a solution of the boundary value problem

$$
\left\{\begin{array}{l}
{ }^{H} D^{\alpha, \beta} x(t)=h(t), \quad t \in[a, b] \\
x(a)=0, \quad \int_{a}^{b} x(s) d s+\mu=\sum_{i=1}^{m-2} \zeta_{i} x\left(\theta_{i}\right),
\end{array}\right.
$$

if and only if

$$
x(t)=I^{\alpha} h(t)+\frac{(t-a)^{\gamma-1}}{\Lambda}\left[\sum_{i=1}^{m-2} \zeta_{i} I^{\alpha} h\left(\theta_{i}\right)-\int_{a}^{b} I^{\alpha} h(s) d s-\mu\right] .
$$

In view of Lemma 3 , we define an operator $\mathcal{A}: C([a, b], \mathbb{R}) \rightarrow C([a, b], \mathbb{R})$ by 


$$
\begin{aligned}
(\mathcal{A} x)(t)= & \frac{(t-a)^{\gamma-1}}{\Lambda}\left(\sum_{i=1}^{m-2} \zeta_{i} I^{\alpha} f\left(s, x(s), I^{\delta} x(s)\right)\left(\theta_{i}\right)-I^{\alpha+1} f\left(s, x(s), I^{\delta} x(s)\right)(b)-\mu\right) \\
& +I^{\alpha} f\left(s, x(s), I^{\delta} x(s)\right)(t), t \in[a, b] .
\end{aligned}
$$

It should be noticed that problem (7) has a solution if and only if the operator $\mathcal{A}$ has fixed points.

3.3.1. Existence and Uniqueness Results for the Problem (7)

Our existence and uniqueness results for the problem (7), based respectively on Banach's contraction mapping principle, Krasnoselskii's fixed point theorem, and the Leray-Schauder nonlinear alternative, are as follows.

Theorem 15. Assume that:

(15.1) there exists a constant $L>0$ such that

$$
\left|f\left(t, x_{1}, x_{2}\right)-f\left(t, y_{1}, y_{2}\right)\right| \leq L\left(\left|x_{1}-y_{1}\right|+\left|x_{2}-y_{2}\right|\right)
$$

for each $t \in[a, b]$ and $x_{i}, y_{i} \in \mathbb{R}, i=1,2$.

If

$$
L_{1}\left\{\frac{(b-a)^{\gamma-1}}{|\Lambda|}\left[\sum_{i=1}^{m-2}\left|\zeta_{i}\right| \frac{\left(\theta_{i}-a\right)^{\alpha}}{\Gamma(\alpha+1)}+\frac{(b-a)^{\alpha+1}}{\Gamma(\alpha+2)}\right]+\frac{(b-a)^{\alpha}}{\Gamma(\alpha+1)}\right\}<1,
$$

where $L_{1}=1+\frac{(b-a)^{\delta}}{\Gamma(\delta+1)}$, then the boundary value problem (7) has a unique solution on $[a, b]$.

Theorem 16. Let $f:[a, b] \times \mathbb{R} \times \mathbb{R} \rightarrow \mathbb{R}$ be a continuous function satisfying (15.1). In addition, we assume that:

(16.1) $|f(t, x, y)| \leq \varphi(t), \quad \forall(t, x, y) \in[a, b] \times \mathbb{R} \times \mathbb{R}$, and $\varphi \in C\left([a, b], \mathbb{R}^{+}\right)$.

Then, the boundary value problem (7) has at least one solution on $[a, b]$ provided

$$
L L_{1} \frac{(b-a)^{\gamma-1}}{|\Lambda|}\left[\sum_{i=1}^{m-2}\left|\zeta_{i}\right| \frac{\left(\theta_{i}-a\right)^{\alpha}}{\Gamma(\alpha+1)}+\frac{(b-a)^{\alpha+1}}{\Gamma(\alpha+2)}\right]<1 .
$$

Theorem 17. Let $f:[a, b] \times \mathbb{R} \times \mathbb{R} \rightarrow \mathbb{R}$ be a continuous function. Assume that:

(17.1) there exist a continuous, nondecreasing, subhomogeneous (that is, $\psi(k x) \leq k \psi(x)$ for all $k \geq 1$ and $\left.x \in \mathbb{R}^{+}\right)$function $\psi:[0, \infty) \rightarrow(0, \infty)$ and a function $p \in C\left([a, b], \mathbb{R}^{+}\right)$such that

$$
|f(t, u, v)| \leq p(t) \psi(|u|+|v|) \quad \text { for each } \quad(t, u, v) \in[a, b] \times \mathbb{R} \times \mathbb{R}
$$

(17.2) there exists a constant $K>0$ such that

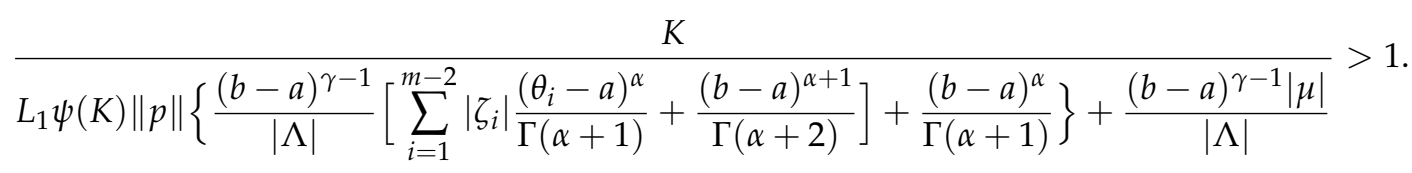

Then, the boundary value problem (7) has at least one solution on $[a, b]$. 
3.3.2. Existence Results for the Inclusion Problem

The corresponding multivalued problem of the form

$$
\left\{\begin{array}{l}
{ }^{H} D^{\alpha, \beta} x(t) \in F\left(t, x(t), I^{\delta} x(t)\right), \quad t \in[a, b], \\
x(a)=0, \quad \int_{a}^{b} x(s) d s+\mu=\sum_{i=1}^{m-2} \zeta_{i} x\left(\theta_{i}\right),
\end{array}\right.
$$

is also studied in [24], where $F:[a, b] \times \mathbb{R}^{2} \rightarrow \mathcal{P}(\mathbb{R})$ is a multivalued map $(\mathcal{P}(\mathbb{R})$ is the family of all nonempty subjects of $\mathbb{R}$ ).

Definition 14. A function $x \in A C([a, b], \mathbb{R})$ is said to be a solution of the problem (8) if there exists a function $v \in L^{1}([a, b], \mathbb{R})$ with $v \in F(t, x)$ a.e. for $t \in[a, b]$ such that $x$ satisfies the differential equation $D^{\alpha} x(t)=v(t)$ for $t \in[a, b]$ and the boundary conditions $x(a)=0, \int_{a}^{b} x(s) d s+\mu=$ $\sum_{i=1}^{m-2} \zeta_{i} x\left(\theta_{i}\right)$.

In the case when $F$ has convex values, existence results based on Martelli's fixed point theorem and the Leray-Schauder nonlinear alternative for multivalued maps, respectively, are given below.

Theorem 18. Assume that the following hypotheses hold:

(18.1) $F:[a, b] \times \mathbb{R} \times \mathbb{R} \rightarrow \mathcal{P}(\mathbb{R})$ is $L^{1}$-Carathéodory;

(18.2) there exists a function $q \in C([a, b], \mathbb{R})$ such that

$$
\|F(t, x, y)\| \leq q(t), \text { for a.e. } t \in[a, b] \text { and each } x, y \in \mathbb{R} .
$$

Then, the problem (8) has at least one solution on $[a, b]$.

Theorem 19. Assume that (18.1) and (17.2) hold. In addition, we assume that:

(19.1) there exists a continuous, nondecreasing, subhomogeneous function $\psi:[0, \infty) \rightarrow(0, \infty)$ and a function $p \in C\left([a, b], \mathbb{R}^{+}\right)$such that

$\|F(t, x, y)\|_{\mathcal{P}}:=\sup \{|y|: y \in F(t, x)\} \leq p(t) \psi(|x|+|y|)$ for each $(t, x, y) \in[a, b] \times$ $\mathbb{R} \times \mathbb{R}$;

Then, the boundary value problem (8) has at least one solution on $[a, b]$.

The existence result for the boundary value problem (8) with a nonconvex-valued right hand side based on the Covitz and Nadler fixed point theorem, is the following.

Theorem 20. Assume that the following conditions hold:

(20.1) $F:[a, b] \times \mathbb{R} \times \mathbb{R} \rightarrow \mathcal{P}_{c p}(\mathbb{R})$ is such that $F(\cdot, x, y):[a, b] \rightarrow \mathcal{P}_{c p}(\mathbb{R})$ is measurable for each $x, y \in \mathbb{R}$;

(20.2) $H_{d}(F(t, x, y), F(t, \bar{x}, \bar{y})) \leq m(t)(|x-\bar{x}|+|y-\bar{y}|)$ for almost all $t \in[a, b]$ and $x, y, \bar{x}, \bar{y} \in$ $\mathbb{R}$ with $m \in C\left([a, b], \mathbb{R}^{+}\right)$, and $d(0, F(t, 0,0)) \leq m(t)$ for almost all $t \in[a, b]$.

Then, the boundary value problem (8) has at least one solution on $[a, b]$ if

$$
L_{1}\left\{\frac{(b-a)^{\gamma-1}}{|\Lambda|}\left[\sum_{i=1}^{m-2}\left|\zeta_{i}\right| \frac{\left(\theta_{i}-a\right)^{\alpha}}{\Gamma(\alpha+1)}+\frac{(b-a)^{\alpha+1}}{\Gamma(\alpha+2)}\right]+\frac{(b-a)^{\alpha}}{\Gamma(\alpha+1)}\right\}\|m\|<1 .
$$

\section{Boundary Value Problems for Sequential Hilfer Fractional Differential Equations and Inclusions}

\subsection{Nonlocal Integro-Multipoint Boundary Conditions}

Existence and uniqueness of solutions were studied in [25] for the following new class of boundary value problems consisting of fractional-order sequential Hilfer-type 
differential equations supplemented with nonlocal integro-multipoint boundary conditions of the form

$$
\left\{\begin{array}{l}
\left({ }^{H} D^{\alpha, \beta}+k^{H} D^{\alpha-1, \beta}\right) x(t)=f\left(t, x(t), I^{\delta} x(t)\right), \quad t \in[a, b] \\
x(a)=0, \quad x(b)=\sum_{i=1}^{m-2} \zeta_{i} I^{\phi_{i}} x\left(\theta_{i}\right)
\end{array}\right.
$$

where ${ }^{H} D^{\alpha, \beta}$ denote the Hilfer fractional derivative operator of order $\alpha, 1<\alpha<2$ and parameter $\beta, 0 \leq \beta \leq 1, f:[a, b] \times \mathbb{R} \times \mathbb{R} \rightarrow \mathbb{R}$ is a continuous function, given constant $k \in \mathbb{R}, I^{\psi}$ as the Riemann-Liouville fractional integral of order $\psi>0, \psi \in\left\{\phi_{i}, \delta\right\}$, $a \geq 0, a<\theta_{1}<\theta_{2}<\cdots<\theta_{m-2}<b$ and $\zeta_{i} \in \mathbb{R}, i=1,2, \ldots, m-2$.

The following lemma concerns a linear variant of the sequential boundary value problem (9).

Lemma 4. Let $h \in C([a, b], \mathbb{R})$ and $a \geq 0,1<\alpha<2, \gamma=\alpha+2 \beta-\alpha \beta$. Assume that

$$
\Lambda:=(b-a)^{\gamma-1}-\Gamma(\gamma) \sum_{i=1}^{m-2} \zeta_{i} \frac{\left(\theta_{i}-a\right)^{\gamma+\phi_{i}-1}}{\Gamma\left(\gamma+\phi_{i}\right)} \neq 0
$$

Then, the function $x$ is a solution of the sequential boundary value problem

$$
\left\{\begin{array}{l}
\left({ }^{H} D^{\alpha, \beta}+k^{H} D^{\alpha-1, \beta}\right) x(t)=f\left(t, x(t), I^{\delta} x(t)\right), \quad t \in[a, b] \\
x(a)=0, \quad x(b)=\sum_{i=1}^{m-2} \zeta_{i} I^{\phi_{i}} x\left(\theta_{i}\right)
\end{array}\right.
$$

if and only if

$$
\begin{aligned}
x(t)= & I^{\alpha} h(t)-k \int_{a}^{t} x(s) d s \\
& +\frac{(t-a)^{\gamma-1}}{\Lambda}\left[\sum_{i=1}^{m-2} \zeta_{i} I^{\alpha+\phi_{i}} h\left(\theta_{i}\right)-k \sum_{i=1}^{m-2} \zeta_{i} I^{\phi_{i}+1} x\left(\theta_{i}\right)+k \int_{a}^{b} x(s) d s-I^{\alpha} h(b)\right] .
\end{aligned}
$$

In view of Lemma 4 , we define an operator $\mathcal{A}: C([a, b], \mathbb{R}) \rightarrow C([a, b], \mathbb{R})$ by

$$
\begin{aligned}
(\mathcal{A} x)(t)= & I^{\alpha} f_{x}^{\delta}(t)-k \int_{a}^{t} x(s) d s \\
& +\frac{(t-a)^{\gamma-1}}{\Lambda}\left[\sum_{i=1}^{m-2} \zeta_{i} I^{\alpha+\phi_{i}} f_{x}^{\delta}\left(\theta_{i}\right)-k \sum_{i=1}^{m-2} \zeta_{i} I^{\phi_{i}+1} x\left(\theta_{i}\right)+k \int_{a}^{b} x(s) d s-I^{\alpha} f_{x}^{\delta}(b)\right],
\end{aligned}
$$

where

$$
f_{x}^{\delta}(t)=f\left(t, x(t), I^{\delta} x(t)\right)
$$

It is obvious that the sequential nonlocal boundary value problem (9) has a solution if and only if the operator $\mathcal{A}$ has fixed points.

We use the following notations:

$$
\Omega=\frac{(b-a)^{\gamma-1}}{|\Lambda|}\left[\sum_{i=1}^{m-2}\left|\zeta_{i}\right| \frac{\left(\theta_{i}-a\right)^{\alpha+\phi_{i}}}{\Gamma\left(\alpha+\phi_{i}+1\right)}+\frac{(b-a)^{\alpha}}{\Gamma(\alpha+1)}\right]+\frac{(b-a)^{\alpha}}{\Gamma(\alpha+1)}
$$

and

$$
\Omega_{1}=\frac{(b-a)^{\gamma-1}}{|\Lambda|}\left[|k| \sum_{i=1}^{m-2}\left|\zeta_{i}\right| \frac{\left(\theta_{i}-a\right)^{\phi_{i}+1}}{\Gamma\left(\phi_{i}+2\right)}+|k|(b-a)\right]+|k|(b-a) .
$$


4.1.1. Existence and Uniqueness Results for the Problem (9)

The existence and uniqueness results for the problem (9) based on Banach's contraction mapping principle, Krasnoselskii's fixed point theorem, and the Leray-Schauder nonlinear alternative, respectively, are given as follows.

Theorem 21. Assume that:

(21.1) there exists a constant $L>0$ such that

$$
\left|f\left(t, x_{1}, x_{2}\right)-f\left(t, y_{1}, y_{2}\right)\right| \leq L\left(\left|x_{1}-y_{1}\right|+\left|x_{2}-y_{2}\right|\right)
$$

for each $t \in[a, b]$ and $x_{i}, y_{i} \in \mathbb{R}, i=1,2$.

If

$$
L L_{1} \Omega+\Omega_{1}<1
$$

where $\Omega$ and $\Omega_{2}$ are defined by (10) and (11), respectively, and $L_{1}=1+\frac{(b-a)^{\delta}}{\Gamma(\delta+1)}$, then the sequential boundary value problem (9) have a unique solution on $[a, b]$.

Theorem 22. Let $f:[a, b] \times \mathbb{R} \times \mathbb{R} \rightarrow \mathbb{R}$ be a continuous function satisfying the following assumption:

(22.1) $|f(t, x, y)| \leq \varphi(t), \quad \forall(t, x, y) \in[a, b] \times \mathbb{R} \times \mathbb{R}$, and $\varphi \in C\left([a, b], \mathbb{R}^{+}\right)$.

Then, if $\Omega_{1}<1$, the sequential boundary value problem (9) has at least one solution on $[a, b]$.

Theorem 23. Assume that $\Omega_{1}<1$ holds. Moreover, we suppose that:

(23.1) there exists a continuous, nondecreasing, subhomogeneous (that is, $\psi(\mu x) \leq \mu \psi(x)$ for all $\mu \geq 1$ and $\left.x \in \mathbb{R}^{+}\right)$function $\psi:[0, \infty) \rightarrow(0, \infty)$ and a function $p \in C\left([a, b], \mathbb{R}^{+}\right)$ such that

$$
|f(t, u, v)| \leq p(t) \psi(|u|+|v|) \quad \text { for each } \quad(t, u, v) \in[a, b] \times \mathbb{R} \times \mathbb{R}
$$

(23.2) there exists a constant $K>0$ such that

$$
\frac{\left(1-\Omega_{1}\right) K}{L_{1} \psi(K)\|p\| \Omega}>1,
$$

where $\Omega$ and $\Omega_{1}$ are defined by (10) and (11), respectively, and $L_{1}$ is defined in Theorem 21. Then, the sequential boundary value problem (9) has at least one solution on $[a, b]$.

4.1.2. Existence Results for the Inclusion Problem

The authors of [25] studied the corresponding multivalued problem

$$
\left\{\begin{array}{l}
\left({ }^{H} D^{\alpha, \beta}+k^{H} D^{\alpha-1, \beta}\right) x(t) \in F\left(t, x(t), I^{\delta} x(t)\right), \quad t \in[a, b], \\
x(a)=0, \quad x(b)=\sum_{i=1}^{m-2} \zeta_{i} I^{\phi_{i}} x\left(\theta_{i}\right),
\end{array}\right.
$$

where $F:[a, b] \times \mathbb{R} \times \mathbb{R} \rightarrow \mathcal{P}(\mathbb{R})$ is a multivalued map and $(\mathcal{P}(\mathbb{R})$ is the family of all nonempty subjects of $\mathbb{R}$ ).

Definition 15. A function $x \in A C([a, b], \mathbb{R})$ is a solution of the sequential boundary value problem (12) if there exists a function $v \in L^{1}([a, b], \mathbb{R})$ with $v \in F(t, x, y)$ a.e. on $[a, b]$ such that $x$ satisfies the sequential fractional differential equation $D^{\alpha} x(t)=v(t)$ on $[a, b]$ and the nonlocal integro-multipoint boundary condition. 
Let us discuss first the case when the multivalued $F$ has convex values. The existence results, based on Martelli's fixed point theorem and the Leray-Schauder nonlinear alternative for multivalued maps, respectively, are the following.

Theorem 24. Assume that $\Omega_{1}<1$ holds. In addition, we assume that:

(24.1) $F:[a, b] \times \mathbb{R} \times \mathbb{R} \rightarrow \mathcal{P}_{c, c p}(\mathbb{R})$ is $L^{1}$-Carathéodory;

(24.2) there exists a function $q \in C([a, b], \mathbb{R})$ such that

$$
\|F(t, x, y)\| \leq q(t), \text { for a.e. } t \in[a, b] \text { and each } x, y \in \mathbb{R} .
$$

Then, the sequential boundary value Problems (12) have at least one solution on $[a, b]$.

Theorem 25. Assume that $\Omega_{1}<1$ and (24.1), (23.2) hold. In addition, we assume that:

(25.1) there exists a continuous, nondecreasing, subhomogeneous function $\psi:[0, \infty) \rightarrow(0, \infty)$ and a function $p \in C\left([a, b], \mathbb{R}^{+}\right)$such that

$$
\|F(t, x, y)\|_{\mathcal{P}}:=\sup \{|y|: y \in F(t, x)\} \leq p(t) \psi(|x|+|y|)
$$

for each $(t, x, y) \in[a, b] \times \mathbb{R} \times \mathbb{R}$.

Then, the sequential boundary value problem (12) has at least one solution on $[a, b]$.

Now, an existence result for the sequential boundary value problem (12) with a possible nonconvex-valued right-hand side, based on the Covitz and Nadler fixed point theorem, is presented.

Theorem 26. Assume that:

(26.1) $F:[a, b] \times \mathbb{R} \times \mathbb{R} \rightarrow \mathcal{P}_{c p}(\mathbb{R})$ is such that $F(\cdot, x, y):[a, b] \rightarrow \mathcal{P}_{c p}(\mathbb{R})$ is measurable for each $x, y \in \mathbb{R}$;

(26.2) $H_{d}(F(t, x, y), F(t, \bar{x}, \bar{y})) \leq m(t)(|x-\bar{x}|+|y-\bar{y}|)$ for almost all $t \in[a, b]$ and $x, y, \bar{x}, \bar{y} \in$ $\mathbb{R}$ with $m \in C\left([a, b], \mathbb{R}^{+}\right)$and $d(0, F(t, 0,0)) \leq m(t)$ for almost all $t \in[a, b]$.

Then, if $L_{1} \Omega\|m\|+\Omega_{1}<1$, the sequential boundary value problem (12) has at least one solution on $[a, b]$.

\subsection{Nonlocal Integro-Multistrip-Multipoint Boundary Conditions}

The authors of [26] studied nonlocal boundary value problems for sequential fractional differential equations involving Hilfer fractional derivatives, supplemented with integromultistrip-multipoint boundary conditions of the form:

$$
\left\{\begin{array}{l}
\left({ }^{H} D^{\alpha, \beta}+k^{H} D^{\alpha-1, \beta}\right) x(t)=f(t, x(t)), \quad t \in[a, b], a \geq 0 \\
x^{(i)}(a)=0, i=0,1,2, \ldots, n-2 \\
\int_{a}^{b} x(s) d s=\sum_{i=2}^{p} \lambda_{i-1} \int_{\eta_{i-1}}^{\eta_{i}} x(s) d s+\sum_{j=1}^{q} \mu_{j} x\left(\rho_{j}\right)
\end{array}\right.
$$

where ${ }^{H} D^{\alpha, \beta}$ denotes the fractional derivative operator of Hilfer type of order $\alpha, n-1<$ $\alpha \leq n$ with $n \geq 3$, and type $\beta, 0 \leq \beta \leq 1, f:[a, b] \times \mathbb{R} \rightarrow \mathbb{R}$ is a continuous function, $a<\eta_{1}<\eta_{2}<\ldots<\eta_{p}<\rho_{1}<\rho_{2}<\ldots<\rho_{q}<b$, and $k, \lambda_{i}, \mu_{j}>0, i=2,3, \ldots, p$, $j=1,2, \ldots, n$ with $p, q \in \mathbb{N}$.

We state an auxiliary lemma that plays a key role to transform the problem (13) into a fixed point problem. 
Lemma 5. Let $h \in C([a, b], \mathbb{R})$ and

$$
\Lambda:=\frac{b-a}{\Gamma(\gamma+1)}-\sum_{i=2}^{p} \lambda_{i-1} \frac{\left[\left(\eta_{i}-a\right)^{\gamma}-\left(\eta_{i-1}-a\right)^{\gamma}\right]}{\Gamma(\gamma+1)}-\sum_{j=1}^{q} \mu_{j} \frac{\left(\rho_{j}-a\right)^{\gamma-1}}{\Gamma(\gamma)} \neq 0 .
$$

Then, $x \in C([a, b], \mathbb{R})$ is a solution of the linear boundary value problem

$$
\left\{\begin{array}{l}
\left({ }^{H} D^{\alpha, \beta}+k{ }^{H} D^{\alpha-1, \beta}\right) x(t)=h(t), \quad t \in[a, b], \\
x^{(i)}(a)=0, \quad i=0,1,2, \ldots, n-2, \\
\int_{a}^{b} x(s) d s=\sum_{i=2}^{p} \lambda_{i-1} \int_{\eta_{i-1}}^{\eta_{i}} x(s) d s+\sum_{j=1}^{q} \mu_{j} x\left(\rho_{j}\right),
\end{array}\right.
$$

if and only if

$$
\begin{aligned}
x(t)= & I^{\alpha} h(t)-k \int_{a}^{t} x(s) d s \\
& +\frac{(t-a)^{\gamma-1}}{\Lambda}\left\{-\int_{a}^{b} I^{\alpha} h(s) d s+\sum_{i=2}^{p} \lambda_{i-1} \int_{\eta_{i-1}}^{\eta_{i}} I^{\alpha} h(s) d s+\sum_{j=1}^{q} \mu_{j} I^{\alpha} h\left(\rho_{j}\right)\right. \\
& \left.-k \sum_{i=2}^{p} \lambda_{i-1} \int_{\eta_{i-1}}^{\eta_{i}} \int_{a}^{s} x(u) d u d s-k \sum_{j=1}^{q} \mu_{j} \int_{a}^{\rho_{j}} x(s) d s+k \int_{a}^{b} \int_{a}^{s} x(u) d u d s\right\} .
\end{aligned}
$$
follows

Having in mind Lemma 5, we introduce an operator $\mathcal{A}: C([a, b], \mathbb{R}) \rightarrow C([a, b], \mathbb{R})$ as

$$
\begin{aligned}
(\mathcal{A} x)(t)= & I^{\alpha} f(t, x(t))-k \int_{a}^{t} x(s) d s+\frac{(t-a)^{\gamma-1}}{\Lambda}\left\{-\int_{a}^{b} I^{\alpha} f(s, x(s)) d s\right. \\
& +\sum_{i=2}^{p} \lambda_{i-1} \int_{\eta_{i-1}}^{\eta_{i}} I^{\alpha} f(s, x(s)) d s+\sum_{j=1}^{q} \mu_{j} I^{\alpha} f\left(\rho_{j}, x\left(\rho_{j}\right)\right) \\
& \left.-k \sum_{i=2}^{p} \lambda_{i-1} \int_{\eta_{i-1}}^{\eta_{i}} \int_{a}^{s} x(u) d u d s-k \sum_{j=1}^{q} \mu_{j} \int_{a}^{\rho_{j}} x(s) d s+k \int_{a}^{b} \int_{a}^{s} x(u) d u d s\right\} .
\end{aligned}
$$

Obviously, the problem (13) is equivalent to the fixed point problem: $x=\mathcal{A} x$.

We use the following notations:

$$
\begin{aligned}
Q= & \frac{(b-a)^{\alpha}}{\Gamma(\alpha+1)}+\frac{(b-a)^{\gamma-1}}{|\Lambda|}\left[\frac{(b-a)^{\alpha+1}}{\Gamma(\alpha+2)}+\sum_{i=2}^{p}\left|\lambda_{i-1}\right| \frac{\left(\eta_{i}-a\right)^{\alpha+1}-\left(\eta_{i-1}-a\right)^{\alpha+1}}{\Gamma(\alpha+2)}\right. \\
& \left.+\sum_{j=1}^{q}\left|\mu_{j}\right| \frac{\left(\rho_{j}-a\right)^{\alpha}}{\Gamma(\alpha+1)}\right]
\end{aligned}
$$

and

$$
\begin{aligned}
Q_{1}= & |k|(b-a)+\frac{(b-a)^{\gamma-1}}{|\Lambda|}\left[|k| \sum_{j=1}^{q}\left|\mu_{j}\right|\left(\rho_{j}-a\right)\right. \\
& \left.+|k| \sum_{i=1}^{p}\left|\lambda_{i-1}\right| \frac{\left(\eta_{i}-a\right)^{2}-\left(\eta_{i-1}-a\right)^{2}}{2}+|k| \frac{(b-a)^{2}}{2}\right] .
\end{aligned}
$$


4.2.1. Existence and Uniqueness Results for the Problem (13)

For the problem (13), we state the following existence and uniqueness results, based on Krasnoselskii's fixed point theorem and Banach's contraction mapping principle, respectively.

Theorem 27. Let $f:[a, b] \times \mathbb{R} \rightarrow \mathbb{R}$ be a continuous function satisfying the conditions:

(27.1) $|f(t, x)-f(t, y)| \leq L|x-y|$, for all $t \in[a, b], L>0, x, y \in \mathbb{R}$;

(27.2) $|f(t, u)| \leq \mu(t)$ for all $(t, u) \in[a, b] \times \mathbb{R}, \mu \in C\left([a, b], \mathbb{R}^{+}\right)$.

Then, the sequential Hilfer fractional boundary value problem (13) has at least one solution on $[a, b]$ provided that $L Q_{1}<1$, where $Q_{1}$ is given by (15).

Theorem 28. Let $f:[a, b] \times \mathbb{R} \rightarrow \mathbb{R}$ be a continuous function satisfying the assumption (27.1). Then, the sequential Hilfer fractional boundary value problem (13) has a unique solution on $[a, b]$ if $L Q+Q_{1}<1$, where $Q$ and $Q_{1}$ are given by (14) and (15), respectively.

\subsubsection{Existence Results for the Inclusion Problem}

The multivalued version of the problem (13) is also studied in [26] by considering the following inclusion problem:

$$
\left\{\begin{array}{l}
\left({ }^{H} D^{\alpha, \beta}+k^{H} D^{\alpha-1, \beta}\right) x(t) \in F(t, x(t)), \quad t \in[a, b], \\
x^{(i)}(a)=0, \quad i=0,1,2, \ldots, n-2, \\
\int_{a}^{b} x(s) d s=\sum_{i=2}^{p} \lambda_{i-1} \int_{\eta_{i-1}}^{\eta_{i}} x(s) d s+\sum_{j=1}^{q} \mu_{j} x\left(\rho_{j}\right),
\end{array}\right.
$$

where $F:[a, b] \times \mathbb{R} \rightarrow \mathcal{P}(\mathbb{R})$ is a multivalued map $(\mathcal{P}(\mathbb{R})$ is the family of all nonempty subsets of $\mathbb{R})$. Now, we consider the multivalued problem (16).

Definition 16. A function $x \in A C([a, b], \mathbb{R})$ is said to be a solution of the problem (16) if there exists a function $v \in L^{1}([a, b], \mathbb{R})$ with $v(t) \in F(t, x)$ a.e. on $[a, b]$ such that

$$
\begin{aligned}
x(t)= & I^{\alpha} v(t)-k \int_{a}^{t} x(s) d s+\frac{(t-a)^{\gamma-1}}{\Lambda}\left\{-\int_{a}^{b} I^{\alpha} v(s) d s\right. \\
& +\sum_{i=2}^{p} \lambda_{i-1} \int_{\eta_{i-1}}^{\eta_{i}} I^{\alpha} v(s) d s+\sum_{j=1}^{q} \mu_{j} I^{\alpha} v\left(\rho_{j}\right)-k \sum_{i=2}^{p} \lambda_{i-1} \int_{\eta_{i-1}}^{\eta_{i}} \int_{a}^{s} x(u) d u d s \\
& \left.-k \sum_{j=1}^{q} \mu_{j} \int_{a}^{\rho_{j}} x(s) d s+k \int_{a}^{b} \int_{a}^{s} x(u) d u d s\right\} .
\end{aligned}
$$

In the first existence result, based on the Leray-Schauder nonlinear alternative for multivalued maps, $F$ has convex values and is $L^{1}$-Carathéodory.

Theorem 29. Assume that $Q_{1}<1$ and the following conditions hold:

(29.1) $F:[a, b] \times \mathbb{R} \rightarrow \mathcal{P}_{c, c p}(\mathbb{R})$ is $L^{1}$-Carathéodory;

(29.2) there exists a continuous, nondecreasing function $\psi:[0, \infty) \rightarrow(0, \infty)$ and a function $p \in C\left([a, b], \mathbb{R}^{+}\right)$such that

$\|F(t, x)\|_{\mathcal{P}}:=\sup \{|y|: y \in F(t, x)\} \leq p(t) \psi(\|x\|)$ for each $(t, x) \in[a, b] \times \mathbb{R} ;$

(29.3) there exists a positive constant $M$ satisfying

$$
\frac{\left(1-Q_{1}\right) M}{\psi(M)\|p\| Q}>1,
$$

where $Q$ and $Q_{1}$ are given by (14) and (15), respectively. 
Then, the sequential Hilfer inclusion fractional boundary value problem (16) has at least one solution on $[a, b]$.

In the second existence result for the problem (16), based on the Covitz and Nadler fixed point theorem, $F$ is a nonconvex-valued multivalued map.

Theorem 30. Assume that the following conditions hold:

(30.1) $F:[a, b] \times \mathbb{R} \rightarrow \mathcal{P}_{c p}(\mathbb{R})$ is such that $F(\cdot, x):[a, b] \rightarrow \mathcal{P}_{c p}(\mathbb{R})$ is measurable for each $x \in \mathbb{R}$;

(30.2) $H_{d}(F(t, x), F(t, \bar{x})) \leq m(t)|x-\bar{x}|$ for almost all $t \in[a, b]$ and $x, \bar{x} \in \mathbb{R}$ with $m \in$ $C\left([a, b], \mathbb{R}^{+}\right)$and $d(0, F(t, 0)) \leq m(t)$ for almost all $t \in[a, b]$.

Then, the sequential Hilfer inclusion fractional boundary value problem (16) has at least one solution on $[a, b]$ if

$$
Q\|m\|+Q_{1}<1,
$$

where $Q$ and $Q_{1}$ are given by (14) and (15), respectively.

\subsection{Riemann-Stieltjes Integral Multistrip Boundary Conditions}

In [27], a new class of sequential Hilfer-type boundary value problems for fractional differential equations involving Riemann-Stieltjes integral multistrip boundary conditions of the form

$$
\left\{\begin{array}{l}
\left({ }^{H} D^{\alpha, \beta}+k^{H} D^{\alpha-1, \beta}\right) x(t)=f(t, x(t)), \quad t \in[a, b] \\
x(a)=0, \quad x(b)=\lambda \int_{a}^{b} x(s) d H(s)+\sum_{i=1}^{n} \mu_{i} \int_{\eta_{i}}^{\xi_{i}} x(s) d s
\end{array}\right.
$$

were discussed, where ${ }^{H} D^{\alpha, \beta}$ denotes the Hilfer fractional derivative operator of order $\alpha, 1<\alpha<2$ and parameter $\beta, 0 \leq \beta \leq 1, f:[a, b] \times \mathbb{R} \rightarrow \mathbb{R}$ is a continuous function, $\int_{a}^{b} x(s) d H(s)$ is the Riemann-Stieltjes integral with respect to the function $H:[a, b] \rightarrow \mathbb{R}$, $a \geq 0, k, \mu_{i} \in \mathbb{R}, a<\eta_{i}<\xi_{i} \leq b, i=1,2, \ldots, n$.

Lemma 6. Let $a \geq 0,1<\alpha<2, \gamma=\alpha+2 \beta-\alpha \beta, h \in C([a, b], \mathbb{R})$, and

$$
\Lambda:=(b-a)^{\gamma-1}-\lambda \int_{a}^{b}(s-a)^{\gamma-1} d H(s)-\frac{1}{\gamma} \sum_{i=1}^{n} \mu_{i}\left[\left(\xi_{i}-a\right)^{\gamma}-\left(\eta_{i}-a\right)^{\gamma}\right] \neq 0 .
$$

Then, the function $x$ is a solution of the sequential boundary value problem

$$
\left\{\begin{array}{l}
\left({ }^{H} D^{\alpha, \beta}+k^{H} D^{\alpha-1, \beta}\right) x(t)=h(t), \quad t \in[a, b], 1<\alpha<2,0 \leq \beta \leq 1, \\
x(a)=0, \quad x(b)=\lambda \int_{a}^{b} x(s) d H(s)+\sum_{i=1}^{n} \mu_{i} \int_{\eta_{i}}^{\xi_{i}} x(s) d s
\end{array}\right.
$$

if and only if

$$
\begin{aligned}
x(t)= & I^{\alpha} h(t)-k \int_{a}^{t} x(s) d s+\frac{(t-a)^{\gamma-1}}{\Lambda}\left[-\lambda \int_{a}^{b}\left[k \int_{a}^{s} x(u) d u-I^{\alpha} h(s)\right] d H(s)\right. \\
& \left.-k \sum_{i=1}^{n} \mu_{i} \int_{\eta_{i}}^{\xi_{i}} \int_{a}^{s} x(u) d u d s+\sum_{i=1}^{n} \mu_{i} \int_{\eta_{i}}^{\xi_{i}} I^{\alpha} h(s) d s+k \int_{a}^{b} x(s) d s-I^{\alpha} h(b)\right] .
\end{aligned}
$$

In view of Lemma 6 , we define an operator $\mathcal{A}: C([a, b], \mathbb{R}) \rightarrow C([a, b], \mathbb{R})$ by

$$
(\mathcal{A} x)(t)=I^{\alpha} f(t, x(t))-k \int_{a}^{t} x(s) d s
$$




$$
\begin{aligned}
& +\frac{(t-a)^{\gamma-1}}{\Lambda}\left[-\lambda \int_{a}^{b}\left[k \int_{a}^{s} x(u) d u-I^{\alpha} f(s, x(s))\right] d H(s)\right. \\
& -k \sum_{i=1}^{n} \mu_{i} \int_{\eta_{i}}^{\xi_{i}} \int_{a}^{s} x(u) d u d s+\sum_{i=1}^{n} \mu_{i} \int_{\eta_{i}}^{\xi_{i}} I^{\alpha} f(s, x(s)) d s \\
& \left.+k \int_{a}^{b} x(s) d s-I^{\alpha} f(b, x(b))\right] .
\end{aligned}
$$

It is obvious that the sequential boundary value problem (17) has solutions if and only if the operator $\mathcal{A}$ has fixed points.

We use the notations:

$$
\begin{aligned}
\Omega= & \frac{(b-a)^{\alpha}}{\Gamma(\alpha+1)}+\frac{(b-a)^{\gamma-1}}{|\Lambda|}\left[|\lambda| \int_{a}^{b} \frac{(s-a)^{\alpha}}{\Gamma(\alpha+1)} d H(s)\right. \\
& \left.+\sum_{i=1}^{n}\left|\mu_{i}\right| \frac{\left(\xi_{i}-a\right)^{\alpha+1}-\left(\eta_{i}-a\right)^{\alpha+1}}{\Gamma(\alpha+2)}+\frac{(b-a)^{\alpha}}{\Gamma(\alpha+1)}\right],
\end{aligned}
$$

and

$$
\begin{aligned}
\Omega_{1}= & |k|(b-a)+\frac{(b-a)^{\gamma-1}}{|\Lambda|}\left[|\lambda||k| \int_{a}^{b}(s-a) d H(s)\right. \\
& \left.+\frac{1}{2}|k| \sum_{i=1}^{n}\left|\mu_{i}\right|\left(\left(\xi_{i}-a\right)^{2}-\left(\eta_{i}-a\right)^{2}\right)+|k|(b-a)\right] .
\end{aligned}
$$

\subsubsection{Existence and Uniqueness Results for the Problem (17)}

The existence and uniqueness results for the problem (17), based on Banach's contraction mapping principle, Krasnoselskii's fixed point theorem, and the Leray-Schauder nonlinear alternative, respectively, are given as follows.

Theorem 31. Assume that:

(31.1) $|f(t, x)-f(t, y)| \leq L|x-y|, L>0, t \in[a, b]$ and $x, y \in \mathbb{R}$.

If

$$
L \Omega+\Omega_{1}<1,
$$

where $\Omega$ and $\Omega_{1}$ are defined by (19) and (20), respectively, then the sequential Hilfer boundary value problem (17) has a unique solution on $[a, b]$.

Theorem 32. Let $f:[a, b] \times \mathbb{R} \rightarrow \mathbb{R}$ be a continuous function such that:

(32.1) $|f(t, w)| \leq \varphi(t), \quad \forall(t, w) \in[a, b] \times \mathbb{R}$, and $\varphi \in C\left([a, b], \mathbb{R}^{+}\right)$.

Then, if $\Omega_{1}<1$, where $\Omega_{1}$ is defined in (19), the sequential Hilfer boundary value problem (17) has at least one solution on $[a, b]$.

Theorem 33. Let $\Omega_{1}<1$. In addition, we assume that:

(33.1) $|f(t, w)| \leq p(t) \psi(|w|)$ for each $(t, w) \in[a, b] \times \mathbb{R}$, where $\psi:[0, \infty) \rightarrow(0, \infty)$ is a nondecreasing and continuous function and $p \in C\left([a, b], \mathbb{R}^{+}\right)$;

(33.2) there exists a constant $K>0$ such that $\frac{\left(1-\Omega_{1}\right) K}{\psi(K)\|p\| \Omega}>1$.

Then, the sequential Hilfer boundary value problem (17) has at least one solution on $[a, b]$. 


\subsubsection{Existence Results for the Inclusion Problem}

The authors of [27] also covered the multivalued case of the problem (17) by considering the following sequential inclusion boundary value problem

$$
\left\{\begin{array}{l}
\left({ }^{H} D^{\alpha, \beta}+k^{H} D^{\alpha-1, \beta}\right) x(t) \in F(t, x(t)), \quad t \in[a, b], \\
x(a)=0, \quad x(b)=\lambda \int_{a}^{b} x(s) d H(s)+\sum_{i=1}^{n} \mu_{i} \int_{\eta_{i}}^{\xi_{i}} x(s) d s
\end{array}\right.
$$

where $F:[a, b] \times \mathbb{R} \rightarrow \mathcal{P}(\mathbb{R})$ is a multivalued function and $(\mathcal{P}(\mathbb{R})$ is the family of all nonempty subsets of $\mathbb{R})$.

Definition 17. A function $x \in A C([a, b], \mathbb{R})$ is a solution of the sequentilal Hilfer boundary value problem (21) if $x(a)=0, x(b)=\lambda \int_{a}^{b} x(s) d H(s)+\sum_{i=1}^{n} \mu_{i} \int_{\eta_{i}}^{\xi_{i}} x(s) d s$, and there exists $a$ function $v \in L^{1}([a, b], \mathbb{R})$ such that $v(t) \in F(t, x(t))$ a.e. on $[a, b]$ and

$$
\begin{aligned}
x(t)= & I^{\alpha} v(t)-k \int_{a}^{t} x(s) d s+\frac{(t-a)^{\gamma-1}}{\Lambda}\left[-\lambda \int_{a}^{b}\left[k \int_{a}^{s} x(u) d u-I^{\alpha} v(s)\right] d H(s)\right. \\
& \left.-k \sum_{i=1}^{n} \mu_{i} \int_{\eta_{i}}^{\xi_{i}} \int_{a}^{s} x(u) d u d s+\sum_{i=1}^{n} \mu_{i} \int_{\eta_{i}}^{\xi_{i}} I^{\alpha} v(s) d s+k \int_{a}^{b} x(s) d s-I^{\alpha} v(b)\right], t \in[a, b] .
\end{aligned}
$$

The following existence result is based on the Leray-Schauder nonlinear alternative for multivalued maps.

Theorem 34. Assume that $\Omega_{1}<1$. In addition, we suppose that:

(34.1) $F:[a, b] \times \mathbb{R} \rightarrow \mathcal{P}_{c, c p}(\mathbb{R})$ is $L^{1}$-Carathéodory multivalued map;

(34.2) $\|F(t, z)\|_{\mathcal{P}}:=\sup \{|y|: y \in F(t, z)\} \leq p(t) \psi(\|z\|)$ for each $(t, z) \in[a, b] \times \mathbb{R}$, where $\psi:[0, \infty) \rightarrow(0, \infty)$ is a nondecreasing continuous function and $p \in C\left([a, b], \mathbb{R}^{+}\right)$;

(34.3) There exists a constant $M>0$ such that

$$
\frac{M}{\|p\| \psi(M) \Omega}>\frac{1}{1-\Omega_{1}},
$$

where $\Omega$ and $\Omega_{1}$ are given in (19) and (20), respectively.

Then, the sequential Hilfer boundary value problem (21) has at least one solution on $[a, b]$.

\section{Boundary Value Problems for $\psi$-Hilfer Fractional Differential Equations}

5.1. Existence Results for a $\psi$-Hilfer Nonlocal Fractional Boundary Value Problem via Topological Degree Theory

The authors of [28], using topological degree theory, studied the existence of solutions for a $\psi$-Hilfer-type fractional differential equation equipped with nonlocal multipoint boundary conditions given by

$$
\left\{\begin{array}{l}
{ }^{H} D_{a^{+}}^{\alpha, \beta ; \psi} x(t)=f(t, x(t)), \quad t \in[a, b], \quad 1<\alpha<2,0 \leq \beta \leq 1 \\
x(a)=0, \quad x(b)-\sum_{i=1}^{m-2} \lambda_{i} x\left(\xi_{i}\right)=\mu h(x)
\end{array}\right.
$$

where ${ }^{H} D_{a^{+}}^{\alpha, \beta ; \psi}$ is the $\psi$-Hilfer fractional derivative of order $\alpha$ and parameter $\beta, a \geq 0$, $f:[a, b] \times \mathbb{R} \rightarrow \mathbb{R}, h: C([a, b], \mathbb{R}) \rightarrow \mathbb{R}$ are given continuous functions, $a<\xi_{1}<\xi_{2}<$ $\ldots<\xi_{m-2}<b$ and $\mu, \lambda_{i} \in \mathbb{R}, i=1,2, \ldots, m-2$.

The following lemma deals with a linear variant of the problem (23). 
Lemma 7. Let $\Lambda \neq 0$. For $z \in C([a, b], \mathbb{R})$, the function $x$ is a solution of the linear problem

$$
\left\{\begin{array}{l}
{ }^{H} D_{a^{+}}^{\alpha, \beta ; \psi} x(t)=z(t), t \in[a, b], a \geq 0,1<\alpha<2,0 \leq \beta \leq 1 \\
x(a)=0, \quad x(b)-\sum_{i=1}^{m-2} \lambda_{i} x\left(\xi_{i}\right)=\mu h(x)
\end{array}\right.
$$

if and only if

$$
x(t)=I^{\alpha} z(t)+\frac{(\psi(t)-\psi(a))^{\gamma-1}}{\Lambda}\left[\mu h(x)-\sum_{i=1}^{m-2} \lambda_{i} I^{\alpha} z\left(\xi_{i}\right)-I^{\alpha} h(b)\right],
$$

where $\gamma=\alpha+2 \beta-\alpha \beta$ and $\Lambda:=(\psi(b)-\psi(a))^{\gamma}-\sum_{i=1}^{m-2} \lambda_{i}\left(\psi\left(\xi_{i}\right)-\psi(a)\right)^{\gamma-1}$.

By Lemma 7 , we introduce an operator $\mathcal{A}: C([a, b], \mathbb{R}) \rightarrow C([a, b], \mathbb{R})$ as

$$
\begin{aligned}
(\mathcal{A} x)(t)= & I^{\alpha} f(t, x(t)) \\
& +\frac{(\psi(t)-\psi(a))^{\gamma-1}}{\Lambda}\left[\mu h(x)+\sum_{i=1}^{m-2} \lambda_{i} I^{\alpha} f\left(\xi_{i}, x\left(\xi_{i}\right)\right)-I^{\alpha} f(b, x(b))\right] .
\end{aligned}
$$

Notice that a fixed point of the operator $\mathcal{A}$ is a solution of the problem (23). We set the following notation:

$$
\begin{aligned}
\delta & =|\mu| \frac{(\psi(b)-\psi(a))^{\gamma-1}}{|\Lambda|} \\
Q & =\frac{(\psi(b)-\psi(a))^{\alpha}}{\Gamma(\alpha+1)}\left(1+\frac{\delta}{|\mu|}\right)+\frac{\delta}{|\mu| \Gamma(\alpha+1)} \sum_{i=1}^{m-2}\left|\lambda_{i}\right|\left(\psi\left(\xi_{i}\right)-\psi(a)\right)^{\alpha}
\end{aligned}
$$

Our existence results are stated below. Theorem 35 is based on Sadovski's fixed point theorem and Theorem 36 on Isaia's fixed point theorem.

Theorem 35. Let $f:[a, b] \times \mathbb{R} \rightarrow \mathbb{R}$ and $h: C([a, b], \mathbb{R}) \rightarrow \mathbb{R}$ be continuous functions such that: (35.1) there exists a constant $L>0$ such that: $|f(t, x)-f(t, y)| \leq L|x-y|$, for all $(t, x),(t, y) \in$ $[a, b] \times \mathbb{R}$

(35.2) there exists a constant $H>0$ such that: $|h(x)-h(y)| \leq H|x-y|$, for all $x, y \in$ $C([a, b], \mathbb{R})$.

Then, there exists at least one solution for the problem (23) if

$$
\delta H+L Q<1
$$

where $\delta$ and $Q$ are defined by (25) and (26), respectively.

Theorem 36. Assume that:

(36.1) for an arbitrary $x \in C([a, b], \mathbb{R})$, there exist $M, N>0, q_{1} \in[0,1)$ such that

$$
|h(x)| \leq M\|x\|^{q_{1}}+N .
$$

(36.2) for an arbitrary $(t, x) \in[a, b] \times \mathbb{R}$, there exist $K, \Xi>0, q_{2} \in[0,1)$ such that

$$
|f(t, x)| \leq K\|x\|^{q_{2}}+\Xi \text {. }
$$

If $\ell=\delta H<1$, then the $\psi$-Hilfer fractional boundary value problem (23) has at least one solution $x \in C([a, b], \mathbb{R})$ and the set of solutions is bounded in $C([a, b], \mathbb{R})$. 


\subsection{Mixed Nonlocal Boundary Conditions}

A new class of boundary value problems of $\psi$-Hilfer fractional integro-differential equations with mixed nonlocal boundary conditions of the form

$$
\left\{\begin{array}{l}
{ }^{H} D_{0^{+}}^{\alpha, \rho ; \psi} x(t)=f\left(t, x(t), I_{0^{+}}^{\phi ; \psi} x(t)\right), \quad t \in(0, T], \\
x(0)=0, \sum_{i=1}^{m} \delta_{i} x\left(\eta_{i}\right)+\sum_{j=1}^{n} \omega_{j} I_{0^{+}}^{\beta_{j} ; \psi} x\left(\theta_{j}\right)+\sum_{k=1}^{r} \lambda_{k}{ }^{H} D_{0^{+}}^{\mu_{k}, \rho ; \psi} x\left(\xi_{k}\right)=\kappa,
\end{array}\right.
$$

were discussed in [29], where ${ }^{H} D_{0^{+}}^{u, p ; \psi}$ is $\psi$-Hilfer fractional derivatives of order $u=\left\{\alpha, \mu_{k}\right\}$ with $1<\mu_{k}<\alpha \leq 2,0 \leq \rho \leq 1, I_{0^{+}}^{v ; \psi}$ is $\psi$-Riemann-Liouville fractional integral of order $v=\left\{\phi, \beta_{j}\right\}, \phi, \beta_{j}>0$ for $j=1,2, \ldots, n, \kappa, \delta_{i}, \omega_{j}, \lambda_{k} \in \mathbb{R}$ are given constants, the points $\eta_{i}, \theta_{j}, \xi_{k} \in[a, b], i=1,2, \ldots, m, j=1,2, \ldots, n, k=1,2, \ldots, r$, and $f:[a, b] \times \mathbb{R}^{2} \rightarrow \mathbb{R}$ is a given continuous function. We emphasize that the mixed nonlocal boundary conditions include multipoint, fractional derivative multiorder, and fractional integral multiorder boundary conditions.

In order to transform the problem (28) into a fixed point problem, we provide the following auxiliary lemma, which concerns a linear variant of the boundary value problem (28).

Lemma 8. Let $1<\mu_{k}<\alpha \leq 2,0 \leq \rho \leq 1, \gamma=\alpha+\rho(2-\alpha), k=1,2, \ldots, r$, and $\Omega \neq 0$. Suppose that $h \in C([a, b], \mathbb{R})$. Then, $x \in C([a, b], \mathbb{R})$ is a solution of the problem

$$
\left\{\begin{array}{l}
{ }^{H} D_{0^{+}}^{\alpha, \rho ; \psi} x(t)=h(t), \quad t \in(0, T], \\
x(0)=0, \quad \sum_{i=1}^{m} \delta_{i} x\left(\eta_{i}\right)+\sum_{j=1}^{n} \omega_{j} I_{0^{+}}^{\beta_{j} ; \psi} x\left(\theta_{j}\right)+\sum_{k=1}^{r} \lambda_{k}{ }^{H} D_{0^{+}}^{\mu_{k}, \rho ; \psi} x\left(\xi_{k}\right)=\kappa,
\end{array}\right.
$$

if and only if $x$ satisfies the integral equation

$$
\begin{aligned}
x(t)= & I_{0^{+}}^{\alpha ; \psi} h(t)+\frac{(\psi(t)-\psi(0))^{\gamma-1}}{\Omega \Gamma(\gamma)}\left[\kappa-\left(\sum_{i=1}^{m} \delta_{i} I_{0^{+}}^{\alpha ; \psi} h\left(\eta_{i}\right)+\sum_{j=1}^{n} \omega_{j} I_{0^{+}}^{\alpha+\beta_{j} ; \psi} h(s)\left(\theta_{j}\right)\right.\right. \\
& \left.\left.+\sum_{k=1}^{r} \lambda_{k} I_{0^{+}}^{\alpha-\mu_{k} ; \psi} h(s)\left(\xi_{k}\right)\right)\right]
\end{aligned}
$$

where

$$
\Omega=\sum_{i=1}^{m} \frac{\delta_{i}\left(\psi\left(\eta_{i}\right)-\psi(0)\right)^{\gamma-1}}{\Gamma(\gamma)}+\sum_{j=1}^{n} \frac{\omega_{j}\left(\psi\left(\theta_{j}\right)-\psi(0)\right)^{\gamma+\beta_{j}-1}}{\Gamma\left(\gamma+\beta_{j}\right)}+\sum_{k=1}^{r} \frac{\lambda_{k}\left(\psi\left(\xi_{k}\right)-\psi(0)\right)^{\gamma-\mu_{k}-1}}{\Gamma\left(\gamma-\mu_{k}\right)} .
$$

For the sake of convenience, we use the following notations:

$$
\begin{aligned}
A(\chi, \varepsilon)= & \frac{(\psi(\chi)-\psi(0))^{\varepsilon}}{\Gamma(\varepsilon+1)} \\
\Lambda_{0}= & 1+A(T, \phi), \\
\Lambda_{1}= & A(T, \alpha)+\frac{A(T, \gamma-1)}{|\Omega|}\left(\sum_{i=1}^{m}\left|\delta_{i}\right| A\left(\eta_{i}, \alpha\right)+\sum_{j=1}^{n}\left|\omega_{j}\right| A\left(\theta_{j}, \alpha+\beta_{j}\right)\right. \\
& \left.+\sum_{k=1}^{r}\left|\lambda_{k}\right| A\left(\xi_{k}, \alpha-\mu_{k}\right)\right) .
\end{aligned}
$$


In view of Lemma 8 , an operator $\mathcal{A}: C([a, b], \mathbb{R}) \rightarrow C([a, b], \mathbb{R})$ is defined by

$$
\begin{aligned}
(\mathcal{A} x)(t)= & I_{0^{+}}^{\alpha ; \psi} F_{x}(s)(t)+\frac{A(t, \gamma-1)}{\Omega}\left[\kappa-\left(\sum_{i=1}^{m} \delta_{i} I_{0^{+}}^{\alpha ; \psi} F_{x}(s)\left(\eta_{i}\right)\right.\right. \\
& \left.\left.+\sum_{j=1}^{n} \omega_{j} I_{0^{+}}^{\alpha+\beta_{j} ; \psi} F_{x}(s)\left(\theta_{j}\right)+\sum_{k=1}^{r} \lambda_{k} I_{0^{+}}^{\alpha-\mu_{k} ; \psi} F_{x}(s)\left(\xi_{k}\right)\right)\right],
\end{aligned}
$$

where

$$
F_{x}(t)=f\left(t, x(t), I_{0^{+}}^{\phi ; \psi} x(t)\right), \quad t \in[a, b] .
$$

It should be noticed that the problem (28) has solutions if and only if the operator $\mathcal{A}$ has fixed points.

In the first result, based on Banach contraction mapping principle, we establish the existence and uniqueness of solutions for the problem (28).

Theorem 37. Assume that:

(37.1) there exists a constant $L_{1}>0$ such that

$$
\left|f\left(t, u_{1}, v_{1}\right)-f\left(t, u_{2}, v_{2}\right)\right| \leq L_{1}\left(\left|u_{1}-u_{2}\right|+\left|v_{1}-v_{2}\right|\right)
$$

for any $u_{i}, v_{i} \in \mathbb{R}, i=1,2$ and $t \in[a, b]$.

If

$$
\Lambda_{0} \Lambda_{1} L_{1}<1
$$

where $\Lambda_{0}$ and $\Lambda_{1}$ are given by (31) and (32), respectively, then the problem (28) has a unique solution on $[a, b]$.

Next, we present two existence results, based on Krasnoselskii's fixed point theorem and the Leray-Schauder nonlinear alternative, respectively.

Theorem 38. Assume that $f:[a, b] \times \mathbb{R}^{2} \rightarrow \mathbb{R}$ is a continuous function satisfying (37.1). In addition, we assume that:

(38.1) $|f(t, u, v)| \leq \sigma(t), \forall(t, u, v) \in[a, b] \times \mathbb{R}^{2}$, and $\sigma \in C\left([a, b], \mathbb{R}^{+}\right)$.

If

$$
L_{1} \Lambda_{0}\left[\Lambda_{1}-A(T, \alpha)\right]<1
$$

where $\Lambda_{0}, \Lambda_{1}, A(T, \alpha)$ are defined by (31), (32), and (30), respectively, then the problem (28) has at least one solution on $[a, b]$.

Theorem 39. Assume that:

(39.1) there exists a function $q \in C\left([a, b], \mathbb{R}^{+}\right)$and a continuous nondecreasing function $\Phi$ : $[0, \infty) \rightarrow[0, \infty)$ that is subhomogeneous (that is, $\Phi(\mu x) \leq \mu \Phi(x)$, for all $\mu \geq 1$ and $x \in C([a, b], \mathbb{R}))$, such that

$$
|f(t, u, v)| \leq q(t) \Phi(|u|+|v|) \quad \text { for each } \quad(t, u, v) \in[a, b] \times \mathbb{R}^{2}
$$

(39.2) there exist a constant $M_{2}>0$ such that

$$
\frac{M_{2}}{\Lambda_{0} \Lambda_{1} \Phi\left(M_{2}\right)\|q\|+(|\kappa| A(T, \gamma-1)) /|\Omega|}>1,
$$

with $\Omega, A(T, \alpha) \Lambda_{0}$ and $\Lambda_{1}$ by (29)-(32).

Then, the problem (28) has at least one solution on $[a, b]$. 


\section{Boundary Value Problems for $\psi$-Hilfer-Type Sequential Fractional Differential Equations and Inclusions \\ 6.1. Multipoint Boundary Conditions}

The study of existence and uniqueness of solutions for a new class of boundary value problems of sequential $\psi$-Hilfer-type fractional differential equations with multipoint boundary conditions of the form

$$
\left\{\begin{array}{l}
\left({ }^{H} D^{\alpha, \beta ; \psi}+{ }^{H}{ }^{H} D^{\alpha-1, \beta ; \psi}\right) x(t)=f(t, x(t)), \quad t \in[a, b], \\
x(a)=0, \quad x(b)=\sum_{i=1}^{m} \lambda_{i} x\left(\theta_{i}\right),
\end{array}\right.
$$

were initiated in [30], where ${ }^{H} D^{\alpha, \beta ; \psi}$ is the $\psi$-Hilfer fractional derivative of order $\alpha$, $1<\alpha<2$ and parameter $\beta, 0 \leq \beta \leq 1, f:[a, b] \times \mathbb{R} \rightarrow \mathbb{R}$ is a continuous function, $a<b, k, \lambda_{i} \in \mathbb{R}, i=1,2, \ldots, m$ and $a<\theta_{1}<\theta_{2}<\ldots<\theta_{m}<b$.

We first give an auxiliary lemma concerning a linear variant of the boundary value problem (33).

Lemma 9. Let $a<b, 1<\alpha<2, \gamma=\alpha+2 \beta-\alpha \beta, h \in C([a, b], \mathbb{R})$, and

$$
\Lambda:=(\psi(b)-\psi(a))^{\gamma-1}-\sum_{i=1}^{m} \lambda_{i}\left(\psi\left(\theta_{i}\right)-\psi(a)\right)^{\gamma-1} \neq 0 .
$$

Then, the function $x \in C([a, b], \mathbb{R})$ is a solution of the boundary value problem

$$
\left\{\begin{array}{l}
\left({ }^{H} D^{\alpha, \beta ; \psi}+k^{H} D^{\alpha-1, \beta ; \psi}\right) x(t)=h(t), \quad t \in[a, b], \\
x(a)=0, \quad x(b)=\sum_{i=1}^{m} \lambda_{i} x\left(\theta_{i}\right)
\end{array}\right.
$$

if and only if

$$
\begin{aligned}
x(t)= & I^{\alpha ; \psi} h(t)-k \int_{a}^{t} x(s) d s+\frac{(\psi(t)-\psi(a))^{\gamma-1}}{\Lambda}\left[-k \sum_{i=1}^{m} \lambda_{i} \int_{a}^{\theta_{i}} x(s) d s\right. \\
& \left.-I^{\alpha ; \psi} h(b)+\sum_{i=1}^{m} \lambda_{i} I^{\alpha ; \psi} h\left(\theta_{i}\right)+k \int_{a}^{b} x(s) d s\right], t \in[a, b] .
\end{aligned}
$$

In view of Lemma 9, we define an operator $\mathcal{A}: C([a, b], \mathbb{R}) \rightarrow C([a, b], \mathbb{R})$ by

$$
\begin{aligned}
(\mathcal{A} x)(t)= & I^{\alpha ; \psi} f(t, x(t))-k \int_{a}^{t} x(s) d s \\
& +\frac{(\psi(t)-\psi(a))^{\gamma-1}}{\Lambda}\left[-k \sum_{i=1}^{m} \lambda_{i} \int_{a}^{\theta_{i}} x(s) d s\right. \\
& \left.+\sum_{i=1}^{m} \lambda_{i} I^{\alpha ; \psi} f\left(\theta_{i}, x\left(\theta_{i}\right)\right)+k \int_{a}^{b} x(s) d s-I^{\alpha ; \psi} f(b, x(b))\right], \quad t \in[a, b] .
\end{aligned}
$$

It should be noticed that the sequential boundary value problem (33) has a solution if and only if the operator $\mathcal{A}$ has fixed points.

In the following, we use the notations:

$$
\Omega=\frac{(\psi(b)-\psi(a))^{\gamma-1}}{|\Lambda| \Gamma(\alpha+1)}\left[\sum_{i=1}^{m}\left|\lambda_{i}\right|\left(\theta_{i}-a\right)^{\alpha}+(\psi(b)-\psi(a))^{\alpha}\right]+\frac{(\psi(b)-\psi(a))^{\alpha}}{\Gamma(\alpha+1)}
$$


and

$$
\Omega_{1}=|k|(b-a)+\frac{(\psi(b)-\psi(a))^{\gamma-1}}{|\Lambda|}\left[|k| \sum_{i=1}^{m}\left|\lambda_{i}\right|\left(\theta_{i}-a\right)+|k|(b-a)\right] .
$$

Our first result is an existence and uniqueness result, and the other two are existence results, based on Banach's contraction mapping principle, Krasnoselskii's fixed point theorem, and the Leray-Schauder nonlinear alternative, respectively.

Theorem 40. Assume that:

(40.1) there exists a constant $L>0$ such that

$$
|f(t, x)-f(t, y)| \leq L|x-y| \text { for each } t \in[a, b] \text { and } x, y \in \mathbb{R} .
$$

If

$$
L \Omega+\Omega_{1}<1,
$$

where $\Omega$ and $\Omega_{1}$ are defined by (34) and (35), respectively, then the boundary value problem (33) has a unique solution on $[a, b]$.

Theorem 41. Let $f:[a, b] \times \mathbb{R} \rightarrow \mathbb{R}$ be a continuous function such that:

(41.1) $|f(t, x)| \leq \varphi(t), \quad \forall(t, x) \in[a, b] \times \mathbb{R}$, and $\varphi \in C\left([a, b], \mathbb{R}^{+}\right)$.

Then, the boundary value problem (33) has at least one solution on $[a, b]$ provided that $\Omega_{1}<1$, where $\Omega_{1}$ is given by (35).

Theorem 42. Let $f:[a, b] \times \mathbb{R} \rightarrow \mathbb{R}$ be a continuous function. Assume that $\Omega_{1}<1$. In addition, we suppose that:

(42.1) there exists a continuous, nondecreasing function $\psi:[0, \infty) \rightarrow(0, \infty)$ and a function $p \in C\left([a, b], \mathbb{R}^{+}\right)$such that

$$
|f(t, u)| \leq p(t) \psi(|u|) \quad \text { for each } \quad(t, u) \in[a, b] \times \mathbb{R} ;
$$

(42.2) there exists a constant $K>0$ such that

$$
\frac{\left(1-\Omega_{1}\right) K}{\psi(K)\|p\| \Omega}>1 .
$$

Then, the boundary value problem (33) has at least one solution on $[a, b]$.

\subsection{Integral Multipoint Boundary Conditions}

The authors of [31] studied a new class of boundary value problems of sequential Hilfer-type fractional differential equations involving integral multipoint boundary conditions of the form

$$
\left\{\begin{array}{l}
\left({ }^{H} D^{\alpha, \beta ; \psi}+k^{H} D^{\alpha-1, \beta ; \psi}\right) x(t)=f(t, x(t)), \quad t \in[a, b], \\
x(a)=0, \quad x(b)=\sum_{i=1}^{n} \mu_{i} \int_{a}^{\eta_{i}} \psi^{\prime}(s) x(s) d s+\sum_{j=1}^{m} \theta_{j} x\left(\xi_{j}\right),
\end{array}\right.
$$

where ${ }^{H} D^{\alpha, \beta ; \psi}$ is the $\psi$-Hilfer fractional derivative operator of order $\alpha, 1<\alpha<2$ and parameter $\beta, 0 \leq \beta \leq 1, k \in \mathbb{R}, f:[a, b] \times \mathbb{R} \rightarrow \mathbb{R}$ is a continuous function, $a \geq 0, \mu_{i}, \theta_{j} \in \mathbb{R}$, $\eta_{i}, \xi_{j} \in(a, b], i=1,2, \ldots, n, j=1,2, \ldots m$, and $\psi$ is a positive increasing function on $(a, b]$, which has a continuous derivative $\psi^{\prime}(t)$ on $(a, b)$.

The following auxiliary lemma concerning a linear variant of the sequential Hilfer boundary value problem (36) plays a fundamental role in establishing the existence and uniqueness results for the given nonlinear problem. 
Lemma 10. Let $a \geq 0,1<\alpha<2,0 \leq \beta \leq 1, \gamma=\alpha+2 \beta-\alpha \beta$ be given constants and

$$
\Lambda:=(\psi(b)-\psi(a))^{\gamma-1}-\frac{1}{\gamma} \sum_{i=1}^{n} \mu_{i}\left(\psi\left(\eta_{i}\right)-\psi(a)\right)^{\gamma}-\sum_{j=1}^{m} \theta_{j}\left(\psi\left(\xi_{j}\right)-\psi(a)\right)^{\gamma-1} \neq 0 .
$$

For a given $h \in C([a, b], \mathbb{R})$, the unique solution of the sequential Hilfer linear fractional boundary value problem

$$
\left\{\begin{array}{l}
\left({ }^{H} D^{\alpha, \beta ; \psi}+k^{H} D^{\alpha-1, \beta ; \psi}\right) x(t)=h(t), \quad t \in[a, b] \\
x(a)=0, \quad x(b)=\sum_{i=1}^{n} \mu_{i} \int_{a}^{\eta_{i}} \psi^{\prime}(s) x(s) d s+\sum_{j=1}^{m} \theta_{j} x\left(\xi_{j}\right)
\end{array}\right.
$$

is given by

$$
\begin{aligned}
x(t)= & I_{a+}^{\alpha ; \psi} h(t)-k \int_{a}^{t} \psi^{\prime}(s) x(s) d s+\frac{(\psi(t)-\psi(a))^{\gamma-1}}{\Lambda}\left[\sum_{i=1}^{n} \mu_{i} \int_{a}^{\eta_{i}} \psi^{\prime}(s) I_{a+}^{\alpha ; \psi} h(s) d s\right. \\
& -k \sum_{i=1}^{n} \mu_{i} \int_{a}^{\eta_{i}} \psi^{\prime}(s) \int_{a}^{s} \psi^{\prime}(u) x(u) d u d s-k \sum_{j=1}^{m} \theta_{j} \int_{a}^{\xi_{i}} \psi^{\prime}(s) x(s) d s \\
& \left.+\sum_{j=1}^{m} \theta_{j} I_{a+}^{\alpha ; \psi} h\left(\xi_{i}\right)+k \int_{a}^{b} \psi^{\prime}(s) x(s) d s-I_{a+}^{\alpha ; \psi} h(b)\right] .
\end{aligned}
$$

Next, in view of Lemma 10 , we define an operator $\mathcal{A}: C([a, b], \mathbb{R}) \rightarrow C([a, b], \mathbb{R})$ by

$$
\begin{aligned}
(\mathcal{A} x)(t)= & I_{a+}^{\alpha ; \psi} f(t, x(t))-k \int_{a}^{t} \psi^{\prime}(s) x(s) d s \\
& +\frac{(\psi(t)-\psi(a))^{\gamma-1}}{\Lambda}\left[-k \sum_{i=1}^{n} \mu_{i} \int_{a}^{\eta_{i}} \psi^{\prime}(s) \int_{a}^{s} \psi^{\prime}(u) x(u) d u d s\right. \\
& +\sum_{i=1}^{n} \mu_{i} \int_{a}^{\eta_{i}} \psi^{\prime}(s) I_{a+}^{\alpha ; \psi} f(s, x(s)) d s-k \sum_{j=1}^{m} \theta_{j} \int_{a}^{\xi_{j}} \psi^{\prime}(s) x(s) d s \\
& \left.+\sum_{j=1}^{m} \theta_{j} I_{a+}^{\alpha ; \psi} f\left(\xi_{j}, x\left(\xi_{j}\right)\right)+k \int_{a}^{b} \psi^{\prime}(s) x(s) d s-I_{a+}^{\alpha ; \psi} f(b, x(b))\right] .
\end{aligned}
$$

In the sequel, we use the following abbreviations:

$$
\begin{aligned}
\Omega= & \frac{(\psi(b)-\psi(a))^{\alpha}}{\Gamma(\alpha+1)}+\frac{(\psi(b)-\psi(a))^{\gamma-1}}{|\Lambda|}\left[\sum_{i=1}^{n}\left|\mu_{i}\right| \frac{\left(\psi\left(\eta_{i}\right)-\psi(a)\right)^{\alpha+1}}{\Gamma(\alpha+2)}\right. \\
& \left.+\sum_{j=1}^{m}\left|\theta_{j}\right| \frac{\left(\psi\left(\xi_{j}\right)-\psi(a)\right)^{\alpha}}{\Gamma(\alpha+1)}+\frac{(\psi(b)-\psi(a))^{\alpha}}{\Gamma(\alpha+1)}\right],
\end{aligned}
$$

and

$$
\begin{aligned}
\Omega_{1}= & |k|(\psi(b)-\psi(a))+\frac{(\psi(b)-\psi(a))^{\gamma-1}}{|\Lambda|}\left[\frac{1}{2}|k| \sum_{i=1}^{n}\left|\mu_{i}\right|\left(\psi\left(\eta_{i}\right)-\psi(a)\right)^{2}\right. \\
& \left.+|k| \sum_{j=1}^{m}\left|\theta_{j}\right|\left(\psi\left(\xi_{j}\right)-\psi(a)\right)+|k|(\psi(b)-\psi(a))\right] .
\end{aligned}
$$

6.2.1. Existence and Uniqueness Results for the Problem (36)

The existence and uniqueness results for the problem (36), based on Banach's contraction mapping principle and Krasnoselskii's fixed point theorem, respectively, are as follows. 
Theorem 43. Assume that:

(43.1) there exists a finite number $L>0$ such that, for all $t \in[a, b]$ and for all $x, y \in \mathbb{R}$, the following inequality is valid:

$$
|f(t, x)-f(t, y)| \leq L|x-y|
$$

Then, the sequential $\psi$-Hilfer fractional boundary value problem (36) has a unique solution on $[a, b]$ provided that

$$
L \Omega+\Omega_{1}<1,
$$

where $\Omega$ and $\Omega_{1}$ are defined by (37) and (38), respectively.

Theorem 44. Let $f:[a, b] \times \mathbb{R} \rightarrow \mathbb{R}$ be a continuous function such that:

(44.1) $|f(t, x)| \leq \varphi(t), \quad \forall(t, x) \in[a, b] \times \mathbb{R}$, and $\varphi \in C\left([a, b], \mathbb{R}^{+}\right)$.

Then, the sequential $\psi$-Hilfer fractional boundary value problem (36) has at least one solution on $[a, b]$ provided that $\Omega_{1}<1$, where $\Omega_{1}$ is defined in (38).

6.2.2. Existence Results for the Inclusion Problem

The multivalued case of the problem (36)

$$
\left\{\begin{array}{l}
\left({ }^{H} D^{\alpha, \beta ; \psi}+k^{H} D^{\alpha-1, \beta ; \psi}\right) x(t) \in F(t, x(t)), \quad t \in[a, b], \\
x(a)=0, \quad x(b)=\sum_{i=1}^{n} \mu_{i} \int_{a}^{\eta_{i}} \psi^{\prime}(s) x(s) d s+\sum_{j=1}^{m} \theta_{j} x\left(\xi_{j}\right),
\end{array}\right.
$$

is also considered in [31], where $F:[a, b] \times \mathbb{R} \rightarrow \mathcal{P}(\mathbb{R})$ is a multivalued function, and $(\mathcal{P}(\mathbb{R})$ is the family of all nonempty subjects of $\mathbb{R})$.

Definition 18. A function $x \in A C([a, b], \mathbb{R})$ is a solution of the problem (39) if $x(a)=0, x(b)=$ $\sum_{i=1}^{n} \mu_{i} \int_{a}^{\eta_{i}} \psi^{\prime}(s) x(s) d s+\sum_{j=1}^{m} \theta_{j} x\left(\xi_{j}\right)$, and there exists a function $v \in L^{1}([a, b], \mathbb{R})$ such that $v(t) \in F(t, x(t))$ a.e. on $[a, b]$ and

$$
\begin{aligned}
x(t)= & I_{a+}^{\alpha ; \psi} v(t)-k \int_{a}^{t} \psi^{\prime}(s) x(s) d s \\
& +\frac{(\psi(t)-\psi(a))^{\gamma-1}}{\Lambda}\left[-k \sum_{i=1}^{n} \mu_{i} \int_{a}^{\eta_{i}} \psi^{\prime}(s) \int_{a}^{s} \psi^{\prime}(u) x(u) d u d s\right. \\
& +\sum_{i=1}^{n} \mu_{i} \int_{a}^{\eta_{i}} \psi^{\prime}(s) I_{a+}^{\alpha ; \psi} v(s) d s-k \sum_{j=1}^{m} \theta_{j} \int_{a}^{\xi_{j}} \psi^{\prime}(s) x(s) d s+\sum_{j=1}^{m} \theta_{j} I_{a+}^{\alpha ; \psi} v\left(\xi_{j}\right) \\
& \left.+k \int_{a}^{b} \psi^{\prime}(s) x(s) d s-I_{a+}^{\alpha ; \psi} v(b)\right]
\end{aligned}
$$

The following existence result is based on the Leray-Schauder nonlinear alternative for multivalued maps.

Theorem 45. Assume that $\Omega_{1}<1$. In addition, we suppose that:

(45.1) $F:[a, b] \times \mathbb{R} \rightarrow \mathcal{P}_{c, c p}(\mathbb{R})$ is $L^{1}$-Carathéodory multivalued map;

(45.2) there exists a nondecreasing and continuous function $\Phi:[0, \infty) \rightarrow(0, \infty)$ and a function $p \in L^{1}\left([a, b], \mathbb{R}^{+}\right)$such that

$$
\|F(t, x)\|_{\mathcal{P}}:=\sup \{|y|: y \in F(t, x)\} \leq p(t) \Phi(\|x\|) \text { for each }(t, x) \in[a, b] \times \mathbb{R}
$$


(45.3) there exists a number $M>0$ such that

$$
\frac{M}{\|p\| \Phi(M) \Omega}>\frac{1}{1-\Omega_{1}},
$$

where $\Omega$ and $\Omega_{1}$ are given in (37) and (38), respectively.

Then, the sequential Hilfer inclusion fractional boundary value problem (39) has at least one solution on $[a, b]$.

\section{Coupled Systems of Hilfer Fractional Differential Equations with Nonlocal Fractional Integral Boundary Conditions}

A new class of coupled systems of Hilfer-type fractional differential equations with nonlocal integral boundary conditions was introduced in [32] by considering the following coupled system

$$
\left\{\begin{array}{l}
{ }^{H} D^{\alpha, \beta} x(t)=f(t, x(t), y(t)), t \in[a, b], \\
{ }^{H} D^{\alpha_{1}, \beta_{1}} y(t)=g(t, x(t), y(t)), \quad t \in[a, b], \\
x(a)=0, \quad x(b)=\sum_{i=1}^{m} \theta_{i} I^{\varphi_{i}} y\left(\xi_{i}\right), \\
y(a)=0, \quad y(b)=\sum_{j=1}^{n} \zeta_{j} I^{\psi_{j}} x\left(z_{j}\right),
\end{array}\right.
$$

where ${ }^{H} D^{\alpha, \beta}$ and ${ }^{H} D^{\alpha_{1}, \beta_{1}}$ are the Hilfer fractional derivatives of orders $\alpha, \alpha_{1}, 1<\alpha, \alpha_{1}<2$, and parameters $\beta, \beta_{1}$, respectively, $0 \leq \beta, \beta_{1} \leq 1$, and $I^{\varphi_{i}}, I^{\psi_{j}}$ are the Riemann-Liouville fractional integrals of order $\varphi_{i}>0$ and $\psi_{j}>0$, respectively, the points $\xi_{i}, z_{j} \in[a, b]$, $a \geq 0, f, g:[a, b] \times \mathbb{R} \times \mathbb{R} \rightarrow \mathbb{R}$ are continuous functions, and $\theta_{i}, \zeta_{j} \in \mathbb{R}, i=1,2, \ldots, m$, $j=1,2, \ldots, n$ are given real constants.

The following lemma deals with a linear variant of the problem (40).

Lemma 11. Let $\varphi_{i}, \psi_{j}>0, \xi_{i}, z_{j} \in[a, b], a \geq 0, \theta_{i}, \zeta_{j} \in \mathbb{R}, i=1,2, \ldots, m, j=1,2, \ldots, n$, $1<\alpha, \alpha_{1}<2,0 \leq \beta, \beta_{1} \leq 1, \gamma=\alpha+2 \beta-\alpha \beta, \gamma_{1}=\alpha_{1}+2 \beta_{1}-\alpha_{1} \beta_{1}, h, h_{1} \in C([a, b], \mathbb{R})$, and

$$
\Lambda=\frac{(b-a)^{\gamma+\gamma_{1}-2}}{\Gamma(\gamma) \Gamma\left(\gamma_{1}\right)}-\left(\sum_{i=1}^{m} \theta_{i} \frac{\left(\xi_{i}-a\right)^{\gamma_{1}+\varphi_{i}-1}}{\Gamma\left(\gamma_{1}+\varphi_{i}\right)}\right)\left(\sum_{j=1}^{n} \zeta_{j} \frac{\left(z_{j}-a\right)^{\gamma+\psi_{j}-1}}{\Gamma\left(\gamma+\psi_{j}\right)}\right) \neq 0 .
$$

Then, the system

$$
\left\{\begin{array}{l}
{ }^{H} D^{\alpha, \beta} x(t)=h(t), \quad t \in[a, b], \\
{ }^{H} D^{\alpha_{1}, \beta_{1}} y(t)=h_{1}(t), \quad t \in[a, b], \\
x(a)=0, \quad x(b)=\sum_{i=1}^{m} \theta_{i} I^{\varphi_{i}} y\left(\xi_{i}\right), \\
y(a)=0, \quad y(b)=\sum_{j=1}^{n} \zeta_{j} I^{\psi_{j}} x\left(z_{j}\right),
\end{array}\right.
$$

is equivalent to the following integral equations

$$
\begin{aligned}
x(t)= & I^{\alpha} h(t)+\frac{(t-a)^{\gamma-1}}{\Lambda \Gamma(\gamma)}\left[\frac{(b-a)^{\gamma_{1}-1}}{\Gamma\left(\gamma_{1}\right)}\left(\sum_{i=1}^{m} \theta_{i} I^{\alpha_{1}+\varphi_{i}} h_{1}\left(\xi_{i}\right)-I^{\alpha} h(b)\right)\right. \\
& \left.+\left(\sum_{i=1}^{m} \theta_{i} \frac{\left(\xi_{i}-a\right)^{\gamma_{1}+\varphi_{i}-1}}{\Gamma\left(\gamma_{1}+\varphi_{i}\right)}\right)\left(\sum_{j=1}^{n} \zeta_{j} I^{\alpha+\psi_{j}} h\left(z_{j}\right)-I^{\alpha_{1}} h_{1}(b)\right)\right]
\end{aligned}
$$


and

$$
\begin{aligned}
y(t)= & I^{\alpha_{1}} h_{1}(t)+\frac{(t-a)^{\gamma_{1}-1}}{\Lambda \Gamma\left(\gamma_{1}\right)}\left[\frac{(b-a)^{\gamma-1}}{\Gamma(\gamma)}\left(\sum_{j=1}^{n} \zeta_{j} I^{\alpha+\psi_{j}} h\left(z_{j}\right)-I^{\alpha_{1}} h_{1}(b)\right)\right. \\
& \left.+\left(\sum_{j=1}^{n} \zeta_{j} \frac{\left(z_{j}-a\right)^{\gamma+\psi_{j}-1}}{\Gamma\left(\gamma+\psi_{j}\right)}\right)\left(\sum_{i=1}^{m} \theta_{i} I^{\alpha_{1}+\varphi_{i}} h_{1}\left(\xi_{i}\right)-I^{\alpha} h(b)\right)\right] .
\end{aligned}
$$

In view of Lemma 11 , we define two operators $\mathcal{K}: C([a, b], \mathbb{R}) \times C([a, b], \mathbb{R}) \rightarrow$ $C([a, b], \mathbb{R}) \times C([a, b], \mathbb{R})$ by

$$
\mathcal{K}(x, y)(t)=\left(\begin{array}{l}
\mathcal{K}_{1}(x, y)(t) \\
\mathcal{K}_{2}(x, y)(t)
\end{array}\right)
$$

where

$$
\begin{aligned}
\mathcal{K}_{1}(x, y)(t)= & I^{\alpha} f_{x, y}(t)+\frac{(t-a)^{\gamma-1}}{\Lambda \Gamma(\gamma)}\left[\frac{(b-a)^{\gamma_{1}-1}}{\Gamma\left(\gamma_{1}\right)}\left(\sum_{i=1}^{m} \theta_{i} I^{\alpha_{1}+\varphi_{i}} g_{x, y}\left(\xi_{i}\right)-I^{\alpha} f_{x, y}(b)\right)\right. \\
& \left.+\left(\sum_{i=1}^{m} \theta_{i} \frac{\left(\xi_{i}-a\right)^{\gamma_{1}+\varphi_{i}-1}}{\Gamma\left(\gamma_{1}+\varphi_{i}\right)}\right)\left(\sum_{j=1}^{n} \zeta_{j} I^{\alpha+\psi_{j}} f_{x, y}\left(z_{j}\right)-I^{\alpha_{1}} g_{x, y}(b)\right)\right],
\end{aligned}
$$

and

$$
\begin{aligned}
\mathcal{K}_{2}(x, y)(t)= & I^{\alpha_{1}} g_{x, y}(t)+\frac{(t-a)^{\gamma_{1}-1}}{\Lambda \Gamma\left(\gamma_{1}\right)}\left[\frac{(b-a)^{\gamma-1}}{\Gamma(\gamma)}\left(\sum_{j=1}^{n} \zeta_{j} I^{\alpha+\psi_{j}} f_{x, y}\left(z_{j}\right)-I^{\alpha_{1}} g_{x, y}(b)\right)\right. \\
& \left.+\left(\sum_{j=1}^{n} \zeta_{j} \frac{\left(z_{j}-a\right)^{\gamma+\psi_{j}-1}}{\Gamma\left(\gamma+\psi_{j}\right)}\right)\left(\sum_{i=1}^{m} \theta_{i} I^{\alpha_{1}+\varphi_{i}} g_{x, y}\left(\xi_{i}\right)-I^{\alpha} f_{x, y}(b)\right)\right],
\end{aligned}
$$

where

$$
f_{x, y}(t)=f(t, x(t), y(t)), g_{x, y}(t)=g(t, x(t), y(t)), \quad t \in[a, b] .
$$

For computational convenience, we set

$$
\begin{aligned}
M_{1}= & \frac{(b-a)^{\alpha}}{\Gamma(\alpha+1)}+\frac{(b-a)^{\gamma-1}}{|\Lambda| \Gamma(\gamma)}\left[\frac{(b-a)^{\gamma_{1}+\alpha-1}}{\Gamma\left(\gamma_{1}\right) \Gamma(\alpha+1)}\right. \\
& \left.+\left(\sum_{i=1}^{m}\left|\theta_{i}\right| \frac{\left(\xi_{i}-a\right)^{\gamma_{1}+\varphi_{i}-1}}{\Gamma\left(\gamma_{1}+\varphi_{i}\right)}\right)\left(\sum_{j=1}^{n}\left|\zeta_{j}\right| \frac{\left(z_{j}-a\right)^{\alpha+\psi_{j}}}{\Gamma\left(\alpha+\psi_{j}+1\right)}\right)\right], \\
M_{2}= & \frac{(b-a)^{\gamma-1}}{|\Lambda| \Gamma(\gamma)}\left[\frac{(b-a)^{\gamma_{1}-1}}{\Gamma\left(\gamma_{1}\right)}\left(\sum_{i=1}^{m}\left|\theta_{i}\right| \frac{\left(\xi_{i}-a\right)^{\alpha_{1}+\varphi_{i}}}{\Gamma\left(\alpha_{1}+\varphi_{i}+1\right)}\right)\right. \\
& \left.+\frac{(b-a)^{\alpha_{1}}}{\Gamma\left(\alpha_{1}+1\right)}\left(\sum_{i=1}^{m}\left|\theta_{i}\right| \frac{\left(\xi_{i}-a\right)^{\gamma_{1}+\varphi_{i}-1}}{\Gamma\left(\gamma_{1}+\varphi_{i}\right)}\right)\right] \\
M_{3}= & \frac{(b-a)^{\gamma_{1}-1}}{|\Lambda| \Gamma\left(\gamma_{1}\right)}\left[\frac{(b-a)^{\gamma-1}}{\Gamma(\gamma)}\left(\sum_{j=1}^{n}\left|\zeta_{j}\right| \frac{\left(z_{j}-a\right)^{\alpha+\psi_{j}}}{\Gamma\left(\alpha+\psi_{j}+1\right)}\right)\right. \\
& \left.+\frac{(b-a)^{\alpha}}{\Gamma(\alpha+1)}\left(\sum_{j=1}^{n}\left|\zeta_{j}\right| \frac{\left(z_{j}-a\right)^{\gamma+\psi_{j}-1}}{\Gamma\left(\gamma+\psi_{j}\right)}\right)\right], \\
M_{4}= & \frac{(b-a)^{\alpha_{1}}}{\Gamma\left(\alpha_{1}+1\right)}+\frac{(b-a)^{\gamma_{1}-1}}{|\Lambda| \Gamma\left(\gamma_{1}\right)}\left[\frac{(b-a)^{\gamma+\alpha_{1}-1}}{\Gamma(\gamma) \Gamma\left(\alpha_{1}+1\right)}\right.
\end{aligned}
$$




$$
\left.+\left(\sum_{j=1}^{n}\left|\zeta_{j}\right| \frac{\left(z_{j}-a\right)^{\gamma+\psi_{j}-1}}{\Gamma\left(\gamma+\psi_{j}\right)}\right)\left(\sum_{i=1}^{m}\left|\theta_{i}\right| \frac{\left(\xi_{i}-a\right)^{\alpha_{1}+\varphi_{i}}}{\Gamma\left(\alpha_{1}+\varphi_{i}+1\right)}\right)\right] .
$$

The existence and uniqueness result, based on Banach's contraction mapping principle, for the system (40) is the following.

Theorem 46. Suppose that $f, g:[a, b] \times \mathbb{R} \times \mathbb{R} \rightarrow \mathbb{R}$ are continuous functions. In addition, we assume that:

(46.1) there exist constants $\ell_{i}, n_{i}, i=1,2$ such that for all $t \in[a, b]$ and $x_{i}, y_{i} \in \mathbb{R}, i=1,2$,

$$
\left|f\left(t, x_{1}, y_{1}\right)-f\left(t, x_{2}, y_{2}\right)\right| \leq \ell_{1}\left|x_{1}-x_{2}\right|+\ell_{2}\left|y_{1}-y_{2}\right|
$$

and

$$
\left|g\left(t, x_{1}, y_{1}\right)-g\left(t, x_{2}, y_{2}\right)\right| \leq n_{1}\left|x_{1}-x_{2}\right|+n_{2}\left|y_{1}-y_{2}\right| .
$$

Then, the system (40) has a unique solution on $[a, b]$, if

$$
\left(M_{1}+M_{3}\right)\left(\ell_{1}+\ell_{2}\right)+\left(M_{2}+M_{4}\right)\left(n_{1}+n_{2}\right)<1 \text {, }
$$

where $M_{i}, i=1,2,3,4$ are given in (41)-(44).

Below are two existence results for the system (40), based on the Leray-Schauder alternative and Krasnoselskii's fixed point theorem, respectively.

Theorem 47. Assume that there exist real constants $u_{i}, v_{i} \geq 0$ for $i=1,2$ and $u_{0}, v_{0}>0$ such that for any $x_{i} \in \mathbb{R},(i=1,2)$, we have:

$$
\begin{aligned}
\left|f\left(t, x_{1}, x_{2}\right)\right| & \leq u_{0}+u_{1}\left|x_{1}\right|+u_{2}\left|x_{2}\right|, \\
\left|g\left(t, x_{1}, x_{2}\right)\right| & \leq v_{0}+v_{1}\left|x_{1}\right|+v_{2}\left|x_{2}\right| .
\end{aligned}
$$

If $\left(M_{1}+M_{3}\right) u_{1}+\left(M_{2}+M_{4}\right) v_{1}<1$ and $\left(M_{1}+M_{3}\right) u_{2}+\left(M_{2}+M_{4}\right) v_{2}<1$, where $M_{i}, i=1,2,3,4$ are given in (41)-(44), then (40) has at least one solution on $[a, b]$.

Theorem 48. Assume that $f, g:[a, b] \times \mathbb{R} \times \mathbb{R} \rightarrow \mathbb{R}$ are continuous functions satisfying assumption (46.1) in Theorem 46. In addition, we suppose that two positive constants $P, Q$ exist such that for all $t \in[a, b]$ and $x_{i}, y_{i} \in \mathbb{R}, i=1,2$,

$$
\left|f\left(t, x_{1}, x_{2}\right)\right| \leq P \text { and }\left|g\left(t, x_{1}, x_{2}\right)\right| \leq Q .
$$

If

$$
\frac{(b-a)^{\alpha}}{\Gamma(\alpha+1)}\left(\ell_{1}+\ell_{2}\right)+\frac{(b-a)^{\alpha_{1}}}{\Gamma\left(\alpha_{1}+1\right)}\left(n_{1}+n_{2}\right)<1,
$$

then the problem (40) has at least one solution on $[a, b]$. 


\section{Coupled Systems of $\psi$-Hilfer Sequential Fractional Differential Equations with Nonlocal Boundary Conditions}

Existence and uniqueness criteria for the solutions of the following nonlocal coupled system of sequential $\psi$-Hilfer fractional derivatives of the form

$$
\begin{cases}\left({ }^{H} D^{\alpha, \beta ; \psi}+k{ }^{H} D^{\alpha-1, \beta ; \psi}\right) x(t)=f(t, x(t), y(t)), & t \in[a, b], \\ \left({ }^{H} D^{p, q ; \psi}+v{ }^{H} D^{p-1, q ; \psi}\right) y(t)=g(t, x(t), y(t)), & t \in[a, b], \\ x(a)=0, \quad x(b)=\sum_{i=1}^{m-2} \lambda_{i} y\left(\theta_{i}\right), & \\ y(a)=0, \quad y(b)=\sum_{j=1}^{n-2} \mu_{j} x\left(\zeta_{j}\right), & \end{cases}
$$

were studied in [33], where ${ }^{H} D^{\alpha, \beta ; \psi}$ and ${ }^{H} D^{p, q ; \psi}$ are the $\psi$-Hilfer fractional derivatives of orders $\alpha$ and $p, 1<\alpha, p \leq 2$, and two parameters $\beta, q, 0 \leq \beta, q \leq 1$, given constants $k, v, \lambda_{i}, \mu_{j} \in \mathbb{R}, a \geq 0$, the points $a<\theta_{1}<\theta_{2}<\cdots<\theta_{m-2}<b, a<\zeta_{1}<\zeta_{2}<\cdots<$ $\zeta_{n-2}<b$, and $f, g:[a, b] \times \mathbb{R} \times \mathbb{R} \rightarrow \mathbb{R}$ are continuous functions.

The following lemma deals with a linear variant of the system (45).

Lemma 12. Let $\gamma=\alpha+2 \beta-\alpha \beta, \delta=p+2 q-p q$, and $h, z \in C([a, b], \mathbb{R})$ be given functions. Then, the unique solution of the $\psi$-Hilfer fractional differential linear system

$$
\left\{\begin{array}{l}
\left({ }^{H} D^{\alpha, \beta ; \psi}+k^{H} D^{\alpha-1, \beta ; \psi}\right) x(t)=h(t), t \in[a, b], \\
\left({ }^{H} D^{p, q ; \psi}+v^{H} D^{p-1, q ; \psi}\right) y(t)=z(t), t \in[a, b], \\
x(a)=0, \quad x(b)=\sum_{i=1}^{m-2} \lambda_{i} y\left(\theta_{i}\right), \\
y(a)=0, \quad y(b)=\sum_{j=1}^{n-2} \mu_{j} x\left(\zeta_{j}\right)
\end{array}\right.
$$

is given by

$$
\begin{aligned}
x(t)= & I^{\alpha ; \psi} h(t)-k I^{1 ; \psi} x(t)+\frac{(\psi(t)-\psi(a))^{\gamma-1}}{\Lambda \Gamma(\gamma)}\left\{\Delta \left[-v \sum_{i=1}^{m-2} \lambda_{i} I^{1 ; \psi} y\left(\theta_{i}\right)\right.\right. \\
& \left.+\sum_{i=1}^{m-2} \lambda_{i} I^{p ; \psi} z\left(\theta_{i}\right)+k I^{1 ; \psi} x(b)-I^{\alpha ; \psi} h(b)\right] \\
& \left.+B\left[-k \sum_{j=1}^{n-2} \mu_{j} I^{1 ; \psi} x\left(\zeta_{j}\right)+\sum_{j=1}^{n-2} \mu_{j} I^{\alpha ; \psi} h\left(\zeta_{j}\right)+v I^{1 ; \psi} y(b)-I^{p ; \psi} z(b)\right]\right\},
\end{aligned}
$$

and

$$
\begin{aligned}
y(t)= & I^{p ; \psi} z(t)-v I^{1 ; \psi} y(t)+\frac{(\psi(t)-\psi(a))^{\delta-1}}{\Lambda \Gamma(\gamma)}\left\{A \left[-k \sum_{j=1}^{n-2} \mu_{j} I^{1 ; \psi} x\left(\zeta_{j}\right)\right.\right. \\
& \left.+\sum_{j=1}^{n-2} \mu_{j} I^{\alpha ; \psi} h\left(\zeta_{j}\right)+v I^{1 ; \psi} y(b)-I^{p ; \psi} z(b)\right] \\
& \left.+\Omega\left[-v \sum_{i=1}^{m-2} \lambda_{i} I^{1 ; \psi} y\left(\theta_{i}\right)+\sum_{i=1}^{m-2} \lambda_{i} I^{p ; \psi} z\left(\theta_{i}\right)+k I^{1 ; \psi} x(b)-I^{\alpha ; \psi} h(b)\right]\right\},
\end{aligned}
$$

where

$$
A=\frac{(\psi(b)-\psi(a))^{\gamma-1}}{\Gamma(\gamma)}, B=\sum_{i=1}^{m-2} \lambda_{i} \frac{\left(\psi\left(\theta_{i}\right)-\psi(a)\right)^{\delta-1}}{\Gamma(\delta)}
$$




$$
\Omega=\sum_{j=1}^{n-2} \mu_{j} \frac{\left(\psi\left(\zeta_{j}\right)-\psi(a)\right)^{\gamma-1}}{\Gamma(\gamma)}, \Delta=\frac{(\psi(b)-\psi(a))^{\delta-1}}{\Gamma(\delta)},
$$

and it is assumed that

$$
\Lambda:=A \Delta-B \Omega \neq 0
$$

In view of Lemma 12, we define an operator $\mathcal{S}: C([a, b], \mathbb{R}) \times C([a, b], \mathbb{R}) \rightarrow C([a, b], \mathbb{R}) \times$ $C([a, b], \mathbb{R})$ by

$$
\mathcal{S}(x, y)(t)=\left(\begin{array}{l}
\mathcal{S}_{1}(x, y)(t) \\
\mathcal{S}_{2}(x, y)(t)
\end{array}\right)
$$

where

$$
\begin{aligned}
& \mathcal{S}_{1}(x, y)(t) \\
= & I^{\alpha ; \psi} f_{x y}(t)-k I^{1 ; \psi} x(t)+\frac{(\psi(t)-\psi(a))^{\gamma-1}}{\Lambda \Gamma(\gamma)}\left\{\Delta \left[-v \sum_{i=1}^{m-2} \lambda_{i} I^{1 ; \psi} y\left(\theta_{i}\right)\right.\right. \\
& \left.+\sum_{i=1}^{m-2} \lambda_{i} I^{p ; \psi} g_{x y}\left(\theta_{i}\right)+k I^{1 ; \psi} x(b)-I^{\alpha ; \psi} f_{x y}(b)\right] \\
& \left.+B\left[-k \sum_{j=1}^{n-2} \mu_{j} I^{1 ; \psi} x\left(\zeta_{j}\right)+\sum_{j=1}^{n-2} \mu_{j} I^{\alpha ; \psi} f_{x y}\left(\zeta_{j}\right)+v I^{1 ; \psi} y(b)-I^{p ; \psi} g_{x y}(b)\right]\right\},
\end{aligned}
$$

and

$$
\begin{aligned}
& \mathcal{S}_{2}(x, y)(t) \\
= & I^{p ; \psi} g_{x y}(t)-v I^{1 ; \psi} y(t)+\frac{(\psi(t)-\psi(a))^{\delta-1}}{\Lambda \Gamma(\gamma)}\left\{A \left[-k \sum_{j=1}^{n-2} \mu_{j} I^{1 ; \psi} x\left(\zeta_{j}\right)\right.\right. \\
& \left.+\sum_{j=1}^{n-2} \mu_{j} I^{\alpha ; \psi} f_{x y}\left(\zeta_{j}\right)+v I^{1 ; \psi} y(b)-I^{p ; \psi} g_{x y}(b)\right] \\
& \left.+\Omega\left[-v \sum_{i=1}^{m-2} \lambda_{i} I^{1 ; \psi} y\left(\theta_{i}\right)+\sum_{i=1}^{m-2} \lambda_{i} I^{p ; \psi} g_{x y}\left(\theta_{i}\right)+k I^{1 ; \psi} x(b)-I^{\alpha ; \psi} f_{x y}(b)\right]\right\},
\end{aligned}
$$

where

$$
f_{x y}(t)=f(t, x(t), y(t)), \text { and } g_{x y}(t)=g(t, x(t), y(t)), t \in[a, b] .
$$

For the sake of computational convenience, we put

$$
\begin{aligned}
& X_{1}=|k|(b-a)+\frac{|A|}{|\Lambda|}|\Delta||k|(b-a)+\frac{|A|}{|\Lambda|}|B||k| \sum_{j=1}^{n-2}\left|\mu_{j}\right|\left(\zeta_{j}-a\right), \\
& Y_{1}=\frac{|A|}{|\Lambda|}|\Delta||v| \sum_{i=1}^{m-2}\left|\lambda_{i}\right|\left(\theta_{i}-a\right)+\frac{|A|}{|\Lambda|}|B| v \mid(b-a), \\
& F_{1}=\frac{(\psi(b)-\psi(a))^{\alpha}}{\Gamma(\alpha+1)}\left(1+\frac{|A|}{|\Lambda|}|\Delta|\right)+\frac{|A|}{|\Lambda|}|B| \sum_{j=1}^{n-2}\left|\mu_{j}\right| \frac{\left(\psi\left(\zeta_{j}\right)-\psi(a)\right)^{\alpha}}{\Gamma(\alpha+1)}, \\
& G_{1}=\frac{|A|}{|\Lambda|}|\Delta| \sum_{i=1}^{m-2}\left|\lambda_{i}\right| \frac{\left(\psi\left(\theta_{i}\right)-\psi(a)\right)^{p}}{\Gamma(p+1)}+\frac{|A|}{|\Lambda|}|B| \frac{(\psi(b)-\psi(a))^{p}}{\Gamma(p+1)}, \\
& X_{2}=\frac{|\Delta|}{|\Lambda|}|A||k| \sum_{j=1}^{n-2}\left|\mu_{j}\right|\left(\zeta_{j}-a\right)+\frac{|\Delta|}{|\Lambda|}|\Omega||k|(b-a), \\
& Y_{2}=|v|(b-a)+\frac{|\Delta|}{|\Lambda|}|A||v|(b-a)+\frac{|\Delta|}{|\Lambda|}|\Omega||v| \sum_{i=1}^{m-2}\left|\lambda_{i}\right|\left(\theta_{i}-a\right),
\end{aligned}
$$




$$
\begin{aligned}
F_{2} & =\frac{|\Delta|}{|\Lambda|}|A| \sum_{j=1}^{n-2}\left|\mu_{j}\right| \frac{\left(\psi\left(\zeta_{j}\right)-\psi(a)\right)^{\alpha}}{\Gamma(\alpha+1)}+\frac{|\Delta|}{|\Lambda|}|\Omega| \frac{(\psi(b)-\psi(a))^{\alpha}}{\Gamma(\alpha+1)} \\
G_{2} & =\frac{(\psi(b)-\psi(a))^{p}}{\Gamma(p+1)}\left(1+\frac{|\Delta|}{|\Lambda|}|A|\right)+\frac{|\Delta|}{|\Lambda|}|\Omega| \sum_{i=1}^{m-2}\left|\lambda_{i}\right| \frac{\left(\psi\left(\theta_{i}\right)-\psi(a)\right)^{p}}{\Gamma(p+1)} .
\end{aligned}
$$

The next theorem describe an existence result, based on the Leray-Schauder alternative, for the system (45).

Theorem 49. Assume that:

(49.1) $f, g:[a, b] \times \mathbb{R} \times \mathbb{R} \rightarrow \mathbb{R}$ are continuous functions and there exist real constants $p_{i}, q_{i} \geq 0,(i=1,2)$ and $p_{0}, q_{0}>0$ such that, $\forall x_{i}, y_{i} \in \mathbb{R},(i=1,2)$,

$$
\left|f\left(t, x_{1}, y_{1}\right)\right| \leq p_{0}+p_{1}\left|x_{1}\right|+p_{2}\left|y_{1}\right|, \quad\left|g\left(t, x_{2}, y_{2}\right)\right| \leq q_{0}+q_{1}\left|x_{2}\right|+q_{2}\left|y_{2}\right| .
$$

If

$$
\begin{aligned}
& \mathcal{M}_{1}=\left[F_{1}+F_{2}\right] p_{1}+\left[G_{1}+G_{2}\right] q_{1}+\left[X_{1}+X_{2}\right]<1, \\
& \mathcal{M}_{2}=\left[F_{1}+F_{2}\right] p_{2}+\left[G_{1}+G_{2}\right] q_{2}+\left[Y_{1}+Y_{2}\right]<1,
\end{aligned}
$$

where $X_{i}, Y_{i}, F_{i}, G_{i}, i=1,2$ are given by (46)-(53), then the system (45) has at least one solution on $[a, b]$.

The uniqueness of solutions of the system (45), based on Banach's contraction mapping principle, is contained in the next theorem.

Theorem 50. Assume that:

(50.1) $f, g:[a, b] \times \mathbb{R} \times \mathbb{R} \rightarrow \mathbb{R}$ are continuous functions and there exist positive constants $\mathcal{P}, \mathcal{Q}$ such that for all $t \in[a, b]$ and $u_{i}, v_{i} \in \mathbb{R}, i=1,2$, we have

$$
\begin{aligned}
& \left|f\left(t, u_{1}, u_{2}\right)-f\left(t, v_{1}, v_{2}\right)\right| \leq \mathcal{P}\left(\left|u_{1}-v_{1}\right|+\left|u_{2}-v_{2}\right|\right), \\
& \left|g\left(t, u_{1}, u_{2}\right)-g\left(t, v_{1}, v_{2}\right)\right| \leq \mathcal{Q}\left(\left|u_{1}-v_{1}\right|+\left|u_{2}-v_{2}\right|\right) .
\end{aligned}
$$

Then, the system (45) has a unique solution on $[a, b]$, provided that

$$
\left[F_{1}+F_{2}\right] \mathcal{P}+\left[G_{1}+G_{2}\right] \mathcal{Q}+\left[X_{1}+X_{2}\right]+\left[Y_{1}+Y_{2}\right]<1,
$$

where $X_{i}, Y_{i}, F_{i}, G_{i}, i=1,2$ are given by (46)-(53).

\section{Existence and Uniqueness of Solutions for System of Hilfer-Hadamard Sequential Fractional Differential Equations with Two Point Boundary Conditions}

The authors of [34] studied the existence and uniqueness of solutions for a new class of system of Hilfer-Hadamard sequential fractional differential equations with two point boundary conditions

$$
\left\{\begin{array}{l}
\left({ }_{H} D_{1^{+}}^{\alpha_{1}, \beta_{1}}+k_{1 H} D_{1+}^{\alpha_{1}-1, \beta_{1}}\right) u(t)=f(t, u(t), v(t)), 1<\alpha_{1} \leq 2, t \in[1, e] \\
\left({ }_{H} D_{1^{+}}^{\alpha_{2}, \beta_{2}}+k_{2 H} D_{1^{+}}^{\alpha_{2}-1, \beta_{2}}\right) v(t)=g(t, u(t), v(t)), 1<\alpha_{2} \leq 2, t \in[1, e] \\
u(1)=0, u(e)=A_{1}, \\
v(1)=0, v(e)=A_{2},
\end{array}\right.
$$

where ${ }_{H} D^{\alpha_{i}, \beta_{i}}$ is the Hilfer-Hadamard fractional derivative of order $\alpha_{i} \in(1,2]$ and type $\beta_{i} \in[0,1]$ for $i \in\{1,2\}, k_{1}, k_{2}, A_{1}, A_{2} \in \mathbb{R}_{+}$, and $f, g:[1, e] \times \mathbb{R} \times \mathbb{R} \rightarrow \mathbb{R}$ are given continuous functions. 
Lemma 13. Let $h_{1}, h_{2} \in C([1, e], \mathbb{R})$. Then, $u, v \in C([1, e], \mathbb{R})$ are solutions of the system of fractional differential equations:

$$
\left\{\begin{array}{l}
\left({ }_{H} D_{1^{+}}^{\alpha_{1}, \beta_{1}}+k_{1 H} D_{1+}^{\alpha_{1}-1, \beta_{1}}\right) u(t)=h_{1}(t), \quad 1<\alpha_{1} \leq 2, t \in[1, e], \\
\left({ }_{H} D_{1^{+}}^{\alpha_{2}, \beta_{2}}+k_{2 H} D_{1^{+}}^{\alpha_{2}-1, \beta_{2}}\right) v(t)=h_{2}(t), \quad 1<\alpha_{2} \leq 2, \quad t \in[1, e],
\end{array}\right.
$$

supplemented with the boundary conditions $u(1)=0, u(e)=A_{1}, v(1)=0, v(e)=A_{2}$ if and only if

$$
\begin{aligned}
u(t)= & A_{1}(\log t)^{\gamma_{1}-1}+k_{1}\left[(\log t)^{\gamma_{1}-1} \int_{1}^{e} \frac{u(s)}{s} d s-\int_{1}^{t} \frac{u(s)}{s} d s\right] \\
& +\frac{1}{\Gamma\left(\alpha_{1}\right)}\left[\int_{1}^{t}\left(\log \frac{t}{s}\right)^{\alpha_{1}-1} \frac{h_{1}(s)}{s} d s-(\log t)^{\gamma_{1}-1} \int_{1}^{e}\left(\log \frac{e}{s}\right)^{\alpha_{1}-1} \frac{h_{1}(s)}{s} d s\right]
\end{aligned}
$$

and

$$
\begin{aligned}
v(t)= & A_{2}(\log t)^{\gamma_{2}-1}+k_{2}\left[(\log t)^{\gamma_{2}-1} \int_{1}^{e} \frac{v(s)}{s} d s-\int_{1}^{t} \frac{v(s)}{s} d s\right] \\
& +\frac{1}{\Gamma\left(\alpha_{2}\right)}\left[\int_{1}^{t}\left(\log \frac{t}{s}\right)^{\alpha_{2}-1} \frac{h_{2}(s)}{s} d s-(\log t)^{\gamma_{2}-1} \int_{1}^{e}\left(\log \frac{e}{s}\right)^{\alpha_{2}-1} \frac{h_{2}(s)}{s} d s\right] .
\end{aligned}
$$

In view of Lemma 13, we define an operator $\mathcal{T}: C([1, e], \mathbb{R}) \times C([1, e], \mathbb{R}) \rightarrow C([1, e], \mathbb{R}) \times$ $C([1, e], \mathbb{R})$ by

$$
\mathcal{T}(u, v)(t)=\left(\mathcal{T}_{1}(u, v)(t), \mathcal{T}_{2}(u, v)(t)\right)
$$

where

$$
\begin{aligned}
\mathcal{T}_{1}(u, v)(t)= & A_{1}(\log t)^{\gamma_{1}-1}+k_{1}\left[(\log t)^{\gamma_{1}-1} \int_{1}^{e} \frac{u(s)}{s} d s-\int_{1}^{t} \frac{u(s)}{s} d s\right] \\
& +\frac{1}{\Gamma\left(\alpha_{1}\right)}\left[\int_{1}^{t}\left(\log \frac{t}{s}\right)^{\alpha_{1}-1} \frac{f(s, u(s), v(s))}{s} d s\right. \\
& \left.-(\log t)^{\gamma_{1}-1} \int_{1}^{e}\left(\log \frac{e}{s}\right)^{\alpha_{1}-1} \frac{f(s, u(s), v(s))}{s} d s\right]
\end{aligned}
$$

and

$$
\begin{aligned}
\mathcal{T}_{2}(u, v)(t)= & A_{2}(\log t)^{\gamma_{2}-1}+k_{2}\left[(\log t)^{\gamma_{2}-1} \int_{1}^{e} \frac{v(s)}{s} d s-\int_{1}^{t} \frac{v(s)}{s} d s\right] \\
& +\frac{1}{\Gamma\left(\alpha_{2}\right)}\left[\int_{1}^{t}\left(\log \frac{t}{s}\right)^{\alpha_{2}-1} \frac{g(s, u(s), v(s))}{s} d s\right. \\
& \left.-(\log t)^{\gamma_{2}-1} \int_{1}^{e}\left(\log \frac{e}{s}\right)^{\alpha_{2}-1} \frac{g(s, u(s), v(s))}{s} d s\right] .
\end{aligned}
$$

The existence and uniqueness results of the system (54), based on the Leray-Schauder alternative and Banach's contraction mapping principle, respectively, are as follows.

Theorem 51. Assume that:

(51.1) There exist real constants $m_{i}, n_{i} \geq 0,(i=1,2)$ and $m_{0}>0, n_{0}>0$, such that for all

$t \in[1, e], x_{i} \in \mathbb{R}, i=1,2$,

$$
\begin{aligned}
& \left|f\left(t, x_{1}, x_{2}\right)\right| \leq m_{0}+m_{1}\left|x_{1}\right|+m_{2}\left|x_{2}\right|, \\
& \left|g\left(t, x_{1}, x_{2}\right)\right| \leq n_{0}+n_{1}\left|x_{1}\right|+n_{2}\left|x_{2}\right| .
\end{aligned}
$$


In addition, it is assumed that $\max \left\{Q_{1}, Q_{2}\right\}<1$ where

$$
Q_{1}:=2\left(k_{1}+\frac{m_{1}}{\Gamma\left(\alpha_{1}+1\right)}+\frac{n_{1}}{\Gamma\left(\alpha_{2}+1\right)}\right), \quad Q_{2}:=2\left(k_{2}+\frac{m_{2}}{\Gamma\left(\alpha_{1}+1\right)}+\frac{n_{2}}{\Gamma\left(\alpha_{2}+1\right)}\right) .
$$

Then, the system (54) has at least one solution on $[1, e]$.

Theorem 52. Assume that:

(52.1) there exist positive constants $L, \bar{L}$ such that for all $t \in[1, e], u_{i}, v_{i} \in \mathbb{R}, i=1,2$,

$$
\begin{aligned}
& \left|f\left(t, u_{1}, u_{2}\right)-f\left(t, v_{1}, v_{2}\right)\right| \leq L\left(\left|u_{1}-v_{1}\right|+\left|u_{2}-v_{2}\right|\right), \\
& \left|g\left(t, u_{1}, u_{2}\right)-g\left(t, v_{1}, v_{2}\right)\right| \leq \bar{L}\left(\left|u_{1}-v_{1}\right|+\left|u_{2}-v_{2}\right|\right) .
\end{aligned}
$$

Then, the system (54) has a unique solution on $[1, e]$, provided that

$$
\mu:=2\left(k_{1}+k_{2}+\frac{L}{\Gamma\left(\alpha_{1}+1\right)}+\frac{\bar{L}}{\Gamma\left(\alpha_{2}+1\right)}\right)<1 .
$$

Funding: This research received no external funding.

Institutional Review Board Statement: Not applicable.

Informed Consent Statement: Not applicable.

Data Availability Statement: Not applicable.

Conflicts of Interest: The author declare no conflict of interest.

\section{References}

1. Diethelm, K. The Analysis of Fractional Differential Equations; Lecture Notes in Mathematics; Springer: New York, NY, USA, 2010.

2. Kilbas, A.A.; Srivastava, H.M.; Trujillo, J.J. Theory and Applications of the Fractional Differential Equations; North-Holland Mathematics Studies; Elsevier Science Inc.: New York, NY, USA, 2006.

3. Lakshmikantham, V.; Leela, S.; Devi, J.V. Theory of Fractional Dynamic Systems; Cambridge Scientific Publishers: Cambridge, UK, 2009.

4. Miller, K.S.; Ross, B. An Introduction to the Fractional Calculus and Differential Equations; John Wiley: NewYork, NY, USA, 1993.

5. Podlubny, I. Fractional Differential Equations; Academic Press: New York, NY, USA, 1999.

6. Samko, S.G.; Kilbas, A.A.; Marichev, O.I. Fractional Integrals and Derivatives; Gordon and Breach Science: Yverdon, Switzerland, 1993.

7. Ahmad, B.; Alsaedi, A.; Ntouyas, S.K.; Tariboon, J. Hadamard-Type Fractional Differential Equations, Inclusions and Inequalities; Springer: Cham, Switzerland, 2017.

8. Ahmad, B.; Ntouyas, S.K. Nonlocal Nonlinear Fractional-Order Boundary Value Problems; World Scientific: Singapore, 2021.

9. Zhou, Y. Basic Theory of Fractional Differential Equations; World Scientific: Singapore, 2014.

10. Hilfer, R. Applications of Fractional Calculus in Physics; World Scientific: Singapore, 2000.

11. Hilfer, R.; Luchko, Y.; Tomovski, Z. Operational method for the solution of fractional differential equations with generalized Riemann-Liouvill fractional derivatives. Frac. Calc. Appl. Anal. 2009, 12, 299-318.

12. Soong, T.T. Random Differential Equations in Science and Engineering; Academic Press: New York, NY, USA, 1973.

13. Almeida, R.; Malinowska, A.B.; Monteiro, M.T.T. Fractional differential equations with a Caputo derivative with respect to a kernel function and their applications. Math Meth Appl Sci. 2017, 41, 336-352. [CrossRef]

14. Hilfer, R. Experimental evidence for fractional time evolution in glass forming materials. J. Chem. Phys. 2002, 284, 399-408. [CrossRef]

15. Hilfer, R. Threefold Introduction to Fractional Derivatives. In Anomalous Transport: Foundations and Applications; Klages, R., Radons, G., Sokolov, I.M., Eds.; Wiley-VCH: Weinheim, Germany, 2008; pp. 17-73.

16. Vanterler da C. Sousa, J.; Capelas de Oliveira, E. Leibniz type rule: $\psi$-Hilfer fractional operator. Commun. Nonlinear Sci. Numer. Simul. 2019, 77, 305-311.

[CrossRef]

17. Vanterler da C. Sousa, J.; Capelas de Oliveira, E. On the $\psi$-Hilfer fractional derivative. Commun. Nonlinear Sci. Numer. Simul. 2018, 60, 72-91. [CrossRef]

18. Qassim, M.D.; Furati, K.M.; Tatar, N.-E. On a differential equation involving Hilfer-hadamard fractional derivative. Abstr. Appl. Anal. 2012, 2012, 391062. [CrossRef]

19. Deimling, K. Multivalued Differential Equations; Walter De Gruyter: Berlin, Germnay; New York, NY, USA, 1992. 
20. Hu, S.; Papageorgiou, N. Handbook of Multivalued Analysis, Volume I: Theory; Kluwer Academic Publishers: Dordrecht, The Netherlands, 1997.

21. Asawasamrit, S.; Kijjathanakorn, A.; Ntouyas, S.K.; Tariboon, J. Nonlocal boundary value problems for Hilfer fractional differential equations. Bull. Korean Math. Soc. 2018, 55, 1639-1657.

22. Wongcharoen, A.; Ntouyas, S.K.; Tariboon, J. Boundary value problems for Hilfer fractional differential inclusions with nonlocal integral boundary conditions. Mathematics 2020, 8, 1905. [CrossRef]

23. Wongcharoen, A.; Ntouyas, S.K.; Tariboon, J. Nonlocal boundary value problems for Hilfer type pantograph fractional differential equations and inclusions. Adv. Differ. Equ. 2020, 2020, 279. [CrossRef]

24. Nuchpong, C.; Ntouyas, S.K.; Tariboon, J. Boundary value problems for Hilfer type fractional integro-differential equations and inclusions with nonlocal integro-multipoint boundary conditions. Open Math. 2020, 18, 1879-1894. [CrossRef]

25. Phuangthong, N.; Ntouyas, S.K.; Tariboon, J.; Nonlaopon, K. Nonlocal sequential boundary value problems for Hilfer type fractional integro-differential equations and inclusions. Mathematics 2021, 9, 615. [CrossRef]

26. Ahmad, B.; Ntouyas, S.K.; Alotaibi, F.M. Boundary value problems for sequential Hilfer fractional differential equations and inclusions with integro-multistrip-multipoint boundary conditions. Fixed Point Theory 2021, accepted for publication.

27. Nuchpong, C.; Ntouyas, S.K.; Samadi, A.; Tariboon, J. Boundary value problems for Hilfer type sequential fractional differential equations and inclusions involving Riemann-Stieltjes integral multi-strip boundary conditions. Adv. Differ. Equ. 2021, $2021,268$. [CrossRef]

28. Ahmad, B.; Ntouyas, S.K.; Alsaedi, A.; Alotaibi, F.M. Existence results for a $\psi$-Hilfer type nonlocal fractional boundary value problem via topological degree theory. Dynam. Systems Appl. 2021, 30, 1091-1103.

29. Sudsutad, W.; Thaiprayoon, C.; Ntouyas, S.K. Existence and stability results for $\psi$-Hilfer fractional integro-differential equation with mixed nonlocal boundary conditions. AIMS Math. 2020, 6, 4119-4141. [CrossRef]

30. Ntouyas, S.K.; Vivek, D. Existence and uniqueness results for sequential $\psi$-Hilfer fractional differential equations with multi-point boundary conditions. Acta Math. Univ. Commen. 2021, XC, 171-185.

31. Sitho, S.; Ntouyas, S.K.; Samadi, A.; Tariboon, J. Boundary value problems for $\psi$-Hilfer type sequential fractional differential equations and inclusions with integral multi-point boundary conditions. Mathematics 2021, 9, 1001. [CrossRef]

32. Wongcharoen, A.; Ntouyas, S.K.; Tariboon, J. On coupled systems for Hilfer fractional differential equations with nonlocal integral boundary conditions. J. Math. 2020, 2020, 2875152. [CrossRef]

33. Wongcharoen, A.; Ntouyas, S.K.; Wongsantisuk, P.; Tariboon, J. Existence results for a nonlocal coupled system of sequential fractional differential equations involving $\psi$-Hilfer fractional derivatives. Adv. Math. Phys. 2021, 2021, 5554619. [CrossRef]

34. Saengthong, W.; Thailert, E.; Ntouyas, S.K. Existence and uniqueness of solutions for system of Hilfer-Hadamard sequential fractional differential equations with two point boundary conditions. Adv. Differ. Equ. 2019, 2019, 525. [CrossRef] 